\title{
On the Massive Sine-Gordon Equation in the First Few Regions of Collapse
}

\author{
G. Benfatto, G. Gallavotti , and F. Nicolò \\ Istituto di Matematica dell'Università di Roma, I-00100 Roma, Italy and \\ Istituto Nazionale di Fisica Nucleare - Sezione di Roma, I-00100 Roma, Italy
}

\begin{abstract}
We study the ultraviolet stability problem for the two-dimensional Yukawa interaction $\lambda \int: \cos \alpha \varphi_{\xi}: d \xi$ in the region $4 \pi<\alpha^{2}<\bar{\alpha}^{2}$, where $6 \pi<\bar{\alpha}^{2}<8 \pi$. The results have a natural Coulomb gas interpretation, because the counterterms do not depend on the field.
\end{abstract}

\section{Introduction}

The cosine potential

$$
V(\varphi)=2 \lambda \int_{\Lambda}: \cos \alpha \varphi_{\xi}: d \xi, \quad \Lambda \subset R^{2}
$$

for the quantized 2-dimensional vibrating string has been widely studied for $\alpha^{2}<4 \pi$ [1], as a perturbation of the elastic string, described in the euclidean formalism by a gaussian random field on $\mathscr{S}^{\prime}\left(R^{2}\right)$ with covariance Kernel

$$
C_{x y}=\frac{1}{(2 \pi)^{2}} \int d k \frac{e^{i k(x-y)}}{1+k^{2}}=\frac{1}{2 \pi} \log \frac{1}{|x-y|}+c+o(|x-y|) .
$$

This theory is equivalent to the classical statistical mechanical problem for the 2-dimensional Yukawa gas treated in the grand canonical ensemble, the activity of the $\pm e$-charges being $\lambda$ and the inverse temperature $\beta$ being such that $\alpha^{2}=\beta e^{2}$. Since the Yukawa potential $C_{x y}$ imitates [see Eq. (1.2)] the Coulomb potential at short distances, the theory in some sense describes the "ultraviolet" part of the Coulomb system. The condition $\alpha^{2}<4 \pi$ is equivalent to the condition that the naive Mayer expansion has finite coefficients. For $\alpha^{2}>4 \pi$ (i.e. low temperature) it is no longer clear that the classical Coulomb system does not collapse. One expects that in fact the gas collapses but that the collapse takes place in a nontrivial and gradual way, while $\alpha^{2}$ varies between $4 \pi$ and $8 \pi$.

A very qualitative and not obviously convincing argument might be the following. Assume that the gas is formed by neutral clusters of $2 n$ particles with

* Partially supported by C.N.R.S. trough the University of Paris VI 
diameter equal to the value $\ell_{N}$ of the ultraviolet cutoff (so that in this situation one would have collapse as $\ell_{N} \rightarrow 0$ ). Then our gas will be formed by independent particles of species $n=1,2, \ldots$, each with activity

$$
\frac{\lambda^{2 n}}{(2 n) !} e^{-\beta \bar{U}^{(N)}}\left(\ell_{N}^{2}\right)^{2 n-1}
$$

where $\bar{U}^{(N)}$ is the cluster energy

$$
\bar{U}^{(N)}=-e^{2}\left[n^{2}-\frac{n(n-1)}{2}-\frac{n(n-1)}{2}\right] C_{0 \ell_{N}}=-\frac{n e^{2}}{2 \pi} \log \ell_{N}^{-1}
$$

and $\left(\ell_{N}^{2}\right)^{2 n-1}$ is the phase space of $(2 n-1)$ particles around the one which defines the cluster's position. The cluster picture will be consistent only if the cluster's activity does not vanish as $N \rightarrow \infty$, i.e. if

$$
\lim _{N \rightarrow \infty}\left[\ell_{N}^{-\left(\frac{\alpha^{2}}{4 \pi}-2\right)}\right]^{2 n} \ell_{N}^{-2}>0 .
$$

So we see that, if $\alpha^{2} \in 8 \pi\left[\frac{2 n-1}{2 n}, \frac{2 n+1}{2(n+1)}\right)$, the maximum cluster size is $2 n$. Hence, if $\alpha^{2}<6 \pi$, the only possible clusters are made of pairs; if $\alpha^{2}<\frac{20 \pi}{3}$ the possible clusters are made with four particles, etc. At $\alpha^{2}=8 \pi$ one should have "full collapse." Since neutral pairs or clusters with infinite activity and zero diameter also represent a collapsed situation, one may think that for $\alpha^{2}<8 \pi$ one has only partial collapse : at the temperatures $\beta_{n}=\frac{8 \pi}{e^{2}} \frac{2 n-1}{2 n} n=1,2, \ldots$, there should be phase transitions where the nature of the "infinite density background" of clusters changes type. This sequence of tresholds was discovered in [1] (Fröhlich).

The above considerations show the interest of the cosine interaction's theory for $\alpha^{2}>4 \pi$ and why it is a theory of the 2 dimensional collapse. In the following sections we shall prove that the above heuristic picture is correct, as far as the stability problem is concerned, at least for $\alpha^{2}<\bar{\alpha}^{2}$, with

$$
\alpha_{4}^{2}<\bar{\alpha}^{2}=2 \pi(\sqrt{17}-1)<\alpha_{6}^{2},
$$

where $\alpha_{4}^{2}=6 \pi$ and $\alpha_{6}^{2}=\frac{20 \pi}{3}$ denote the thresholds for the appearance of the fourparticles and six-particles neutral clusters [see Eq. (1.5)]. The value that we find for $\bar{\alpha}^{2}$ has probably no special meaning and it may be an artifact of our estimate techniques, see comment after (B.12).

\section{Nonperturbative Renormalization}

The cutoff field $\varphi_{\xi}^{(N)}, \xi \in R^{2}$ will be defined, as in [2], as the gaussian field with covariance

$$
C_{N}=(1-D)^{-1}-\left(\gamma^{2 N+2}-D\right)^{-1},
$$


where $D$ is the Laplace operator in $R^{2}$ and $\gamma>1$ is a constant which will be chosen later suitably close to $1 . \varphi_{\xi}^{(N)}$ can be represented as a sum of independent, identically distributed up to scale factors, gaussian fields

$$
\tilde{\varphi}_{\xi}^{(h)}, k=0,1, \ldots, N \mid, \quad \varphi_{\xi}^{(N)}=\sum_{0}^{N} \hat{k} \tilde{\varphi}_{\xi}^{(k)},
$$

where, by definition, $\tilde{\varphi}_{\xi}^{(k)}$ is the gaussian field with covariance:

$$
\tilde{C}_{k}(\xi-\eta)=\frac{1}{(2 \pi)^{2}} \int d p e^{i p(\xi-\eta)}\left(\frac{1}{\gamma^{2 k}+p^{2}}-\frac{1}{\gamma^{2 k+2}+p^{2}}\right) .
$$

We call the "field of frequency $k$ " the field $\tilde{\varphi}^{(k)} . P_{k}$ and $\tilde{P}_{k}\left(\mathscr{E}_{k}\right.$ and $\left.\tilde{\mathscr{E}}_{k}\right)$ will denote the measures (expectations) corresponding to the fields $\varphi^{(k)}$ and $\tilde{\varphi}^{(k)}$, respectively.

Observe the scaling relation

$$
\tilde{C}_{k}(\xi-\eta)=\tilde{C}_{0}\left(\gamma^{k} \xi-\gamma^{k} \eta\right)
$$

which implies, in particular, that the fields

$$
z_{\xi}^{(k)}=\tilde{\varphi}_{\gamma^{-k} \xi}^{(k)}
$$

are identically and independently distributed with covariance $\tilde{C}_{0}(\xi-\eta)$. If $I$ is a cube centered at the origin, we define: ${ }^{1}$

$$
V_{0, I}^{(N)}=2 \lambda \int_{I}: \cos \alpha \varphi_{\xi}^{(N)}: d \xi
$$

As a function of $z_{\xi}^{(N)}, V_{0, I}^{(N)}$ can be written in the following way [using (2.5)]

$$
V_{0, I}^{(N)}=2 \lambda_{N} \int_{\gamma^{N} I} \cos \alpha\left(\varphi_{\gamma-N \xi}^{(N-1)}+z_{\xi}^{(N)}\right) d \xi
$$

where

$$
\lambda_{N}=\lambda e^{\frac{\alpha^{2}}{2} C_{N}(0)} \gamma^{-2 N}=\lambda \gamma^{\frac{\alpha^{2}}{4 \pi}} \gamma^{\left(\frac{\alpha^{2}}{4 \pi}-2\right) N}
$$

will be called "effective coupling constant" for the frequency $N$. From Eq. (2.8) follows that the theory is exponentially asymptotically free if $\alpha^{2}<8 \pi$. For these values of $\alpha^{2}$ we can then hope that the renormalization group analysis used in [2] for the $\Phi_{3}^{4}$ theory works. However for $\alpha^{2} \geqq 4 \pi$ the theory with interaction $V_{0, I}^{(N)}$ is divergent in the limit $N \rightarrow \infty$ and we need to add some counter-terms in order to regularize it. Call $V_{I}^{(N)}$ the renormalized interaction and define

$$
\begin{aligned}
\tilde{V}_{I}^{(h)} & =\left[\sum_{0}^{t} \frac{1}{n !} \tilde{\mathscr{E}}_{h+1}^{T}\left(\tilde{V}_{I}^{(h+1)} ; n\right)\right]_{t} \quad h=-1,0, \ldots, N-1, \\
\tilde{V}_{I}^{(N)} & =V_{I}^{(N)},
\end{aligned}
$$

where $\tilde{\mathscr{E}} T h(\cdot ; n)$ is the truncated expectation of order $n^{2}$ with respect to the measure $\tilde{P}_{h}$ and $\left[\sum_{n} c_{n} \lambda^{n}\right]_{t}$ is the truncation to order $t$ of the polynomial $\sum_{n} c_{n} \lambda^{n}$. Suppose now

1 As usual, if $x$ is a random variable: : $\cos x:=e^{\frac{1}{2}\left\langle x^{2}\right\rangle} \cos x$.

2 If $f_{1}, \ldots, f_{n}$ are random variables we define

$$
\mathscr{E}^{T}\left(f_{1}, \ldots, f_{n}\right)=\frac{\partial^{n}}{\partial \lambda_{1}, \ldots, \partial \lambda_{n}} \log \mathscr{E}\left(e^{\sum_{1}^{n} \lambda_{2} f_{1}}\right)
$$

where $\mathscr{E}$ denotes the expectation. If $f_{1}=f_{2}=\ldots=f_{n}=f$, we put also $\mathscr{E}^{T}(f, n)=\mathscr{E}^{T}(f, f, \ldots, f)$ 
that it is possible to choose the counterterms so that $\tilde{V}_{I}^{(h)}$ as a function of $z^{(h)}$, is a polynomial in $\lambda_{h}$ with coefficients weakly dependent on $h$, at least for a set of values of $\varphi^{(h-1)}$ of large probability. Then simple heuristic considerations suggest that, in some sense:

with

$$
\hat{\mathscr{E}}_{h+1}\left(e^{\tilde{V}_{I}^{(h+1)}}\right)=\exp \left(\tilde{V}_{I}^{(h)}+R_{I}^{(h)}\right)
$$

$$
R_{I}^{(h)} \sim \lambda_{h}^{t+1} \gamma^{2 h}|I|
$$

This implies that we can hope to estimate the partition function

$$
Z_{N}(I)=\mathscr{E}_{N}\left(e^{V_{I}^{(N)}}\right)
$$

by successively integrating over $z^{(N)}, z^{(N-1)}, \ldots, z^{(0)}$ only if the right hand side of Eq. (2.11) is summable in $h$ that is only if [see Eq. (2.8)]

$$
\left(\frac{\alpha^{2}}{4 \pi}-2\right)(t+1)+2<0
$$

These considerations also suggest that we need counterterms of order at most $\left[2 /\left(2-\frac{\alpha^{2}}{4 \pi}\right)\right]$. It turns out that, if $\alpha^{2}<\bar{\alpha}^{2}$ [see Eq. (1.6)], we can satisfy the conditions on $\tilde{V}_{I}^{(h)}$ by adding to $V_{0, I}^{(N)}$ only constant counterterms associated in the Coulomb gas interpretation at the collapsing clusters. This is very important since the presence of counterterms depending on the field would destroy the relation between the renormalized theory and the Coulomb gas problem.

The idea of the possibility of a complete renormalization by the use of constant counterterms only is not new : we learned it from Fröhlich. For the massless case see [5].

We conjecture that it is possible to renormalize the cosine interaction by adding only constant counterterms for all $\alpha^{2}<8 \pi$. However we could not prove that much, for reasons which will become clear in the following and which seem essentially technical. We therefore assume the condition on $\alpha^{2}$ :

$$
4 \pi \leqq \alpha^{2}<\bar{\alpha}^{2}=2 \pi(\sqrt{17}-1)<\frac{32 \pi}{5} .
$$

In this case we can choose in Eq. (2.9) $t=4$ and we define

$$
V_{I}^{(N)}=V_{0, I}^{(N)}-\frac{1}{2 !} \mathscr{E}_{N}^{T}\left(V_{0, I}^{(N)} ; 2\right)-\frac{1}{4 !} \mathscr{E}_{N}^{T}\left(V_{0, I}^{(N)} ; 4\right) .
$$

We are interested in proving the existence of the limit.

$$
\lim _{N \rightarrow \infty} \mathscr{E}_{N}\left(\exp \left(V_{I}^{(N)}+\varphi^{(N)}(f)\right)\right) \equiv Z_{I}(f)
$$

for $f \in D\left(R^{2}\right), \varphi^{(N)}(f)=\int \varphi_{\xi}^{(N)} f(\xi) d \xi$.

The main part of the proof will be the following theorem.

Theorem 1. There exist two constants $E_{+}(\lambda), E_{-}(\lambda)$ such that, uniformly in $N$ :

$$
\exp \left[-E_{-}(\lambda)|I|\right] \leqq \mathscr{E}_{N}\left(\exp \bar{V}_{I}^{(N)}\right) \leqq \exp \left[E_{+}(\lambda)|I|\right]
$$


where $|I|$ is the volume and

$$
\bar{V}_{I}^{(N)}=V_{I}^{(N)}-\mathscr{E}_{N}\left(V_{0, I}^{(N)}\right)-\frac{1}{3 !} \mathscr{E}_{N}^{T}\left(V_{0, I}^{(N)} ; 3\right)
$$

Furthermore, $\forall \varepsilon^{\prime}>0$ :

$$
\begin{gathered}
\lim _{\lambda \rightarrow 0} \lambda^{-5+\varepsilon^{\prime}}\left|E_{-}(\lambda)\right|=0, \\
\lim _{\lambda \rightarrow 0} \lambda^{-4}\left|E_{+}(\lambda)\right|=0 .
\end{gathered}
$$

Observe that $\bar{V}_{I}^{(N)}$ differs from $V_{I}^{(N)}$ by a constant finite in the limit $N \rightarrow \infty$.

The proof that $Z_{I}(f)$ exists will easily follow from the proof of Theorem 1, which will be given in the following sections.

\section{The Structure of $\tilde{V}_{I}^{(h)}$}

In this section we shall collect the properties of $\tilde{V}_{I}^{(h)}$ that will be important in the following.

$\tilde{V}_{I}^{(h)}$ has the following structure [if $t=4$ in Eq. (2.9)], as we show in Appendix A:

$$
\tilde{V}_{I}^{(h)}=V_{0, I}^{(h)}-\lambda_{h}^{2} W_{I^{2}}^{(2, h)}+\lambda_{h}^{3} \sum_{1}^{2} W_{I^{2} \times I}^{(3, i, h)}+\lambda_{h}^{4} \sum_{i}^{7} W_{I^{2} \times I^{2}}^{(4, i, h)}+A_{I}^{(h)}+C_{I}^{(h)},
$$

where, for $F, G \subset R^{2}$ and $J \subset R$ arbitrary bounded sets ${ }^{3}$ :

$$
\begin{aligned}
& W_{F}^{(2, h)}=\int_{F} d \xi_{1} d \xi_{2} u_{N}^{(2, h)}\left(\xi_{1}, \xi_{2}\right) \frac{:\left[1-\cos \left(\alpha \varphi_{\xi_{1}}^{(h)}-\alpha \varphi_{\xi_{2}}^{(h)}\right)\right]:}{\left(\gamma^{h}\left|\xi_{1}-\xi_{2}\right|\right)^{2(1-\varepsilon)}}, \\
& W_{F \times J}^{(3,1, h)}=\int_{F \times J} d \xi_{1} d \xi_{2} d \xi_{3} u_{N}^{(3,1, h)}\left(\xi_{1}, \xi_{2}, \xi_{3}\right) \cos \left(\alpha \varphi_{\xi_{1}}^{(h)}-\alpha \varphi_{\xi_{2}}^{(h)}\right) \cos \alpha \varphi_{\xi_{3}}^{(h)}, \\
& W_{F \times J}^{(3,2, h)}=\int_{F \times J} d \xi_{1} d \xi_{2} d \xi_{3} u_{N}^{(3,2, h)}\left(\xi_{1}, \xi_{2}, \xi_{3}\right) \frac{\sin \left(\alpha \varphi_{\xi_{1}}^{(h)}-\alpha \varphi_{\xi_{2}}^{(h)}\right) \sin \alpha \varphi_{\xi_{3}}^{(h)}}{\left(\gamma^{h}\left|\xi_{1}-\xi_{2}\right|\right)^{1-\varepsilon}}, \\
& W_{F \times G}^{(4,1, h)}=\int_{F \times G} d \xi_{1} d \xi_{2} d \xi_{3} d \xi_{4} u_{N}^{(4,1, h)}(\xi) \\
& :\left[\frac{1-\cos \left(\alpha \varphi_{\xi 1}^{(h)}-\alpha \varphi_{\xi 2}^{(h)}\right)}{\left(\gamma^{h}\left|\xi_{1}-\xi_{2}\right|\right)^{2(1-\varepsilon)}} \cos \left(\alpha \varphi_{\xi_{3}}^{(h)}-\alpha \varphi_{\xi_{4}}^{(h)}\right)\right]: \\
& W_{F \times G}^{(4,2, h)}=\int_{F \times G} d \hat{\xi} u_{N}^{(4,2, h)}(\underline{\xi}) \frac{:\left[1-\cos \left(\alpha \varphi_{\xi_{3}}^{(h)}-\alpha \varphi_{\xi_{4}}^{(h)}\right)\right]:}{\left(\gamma^{h}\left|\xi_{3}-\xi_{4}\right|\right)^{2(1-\varepsilon)}}, \\
& W_{F \times G}^{(4,3, h)}=\int_{F \times G} d \underline{\xi} u_{N}^{(4,3, h)}(\underline{\xi}):\left[\frac{\sin \left(\alpha \varphi_{\xi_{1}}^{(h)}-\alpha \varphi_{\xi_{2}}^{(h)}\right)}{\left(\gamma^{h}\left|\xi_{1}-\xi_{2}\right|\right)^{1-\varepsilon}} \frac{\sin \left(\alpha \varphi_{\xi_{3}}^{(h)}-\alpha \varphi_{\xi_{4}}^{(h)}\right)}{\left(\gamma^{h}\left|\xi_{3}-\xi_{4}\right|\right)^{1-\varepsilon}}\right]:, \\
& W_{F \times G}^{(4,4, h)}=\int_{F \times G} d \underline{\xi} u_{N}^{(4,4, h)}(\xi):\left[\frac{1-\cos \left(\alpha \varphi_{\xi_{1}}^{(h)}-\alpha \varphi_{\xi_{2}}^{(h)}\right)}{\left(\gamma^{h}\left|\xi_{1}-\xi_{2}\right|\right)^{2(1-\varepsilon)}} \cos \left(\alpha \varphi_{\xi_{3}}^{(h)}-\alpha \varphi_{\xi_{4}}^{(h)}\right)\right]: \text {, }
\end{aligned}
$$

3 In the following expressions one sees terms, like (3.5) and (3.8) or (3.6) and (3.9), which look the same: however they arise in a different way and they must be bounded through different estimates (see Appendix A) 


$$
\begin{aligned}
W_{F \times G}^{(4,5, h)}= & \int_{F \times G} d \underline{\xi_{1}} u_{N}^{(4,5, h)}(\underline{\xi}) \frac{:\left[1-\cos \left(\alpha \varphi_{\xi_{3}}^{(h)}-\alpha \varphi_{\xi_{4}}^{(h)}\right)\right]:}{\left(\gamma^{h}\left|\xi_{3}-\xi_{4}\right|\right)^{2(1-\varepsilon)}}, \\
W_{F \times G}^{(4,6, h)=} & \int_{F \times G} d \underline{\xi} u_{N}^{(4,6, h)}(\underline{\xi}) \cos \left(\alpha \varphi_{\xi_{1}}^{(h)}-\alpha \varphi_{\xi_{2}}^{(h)}\right) \cos \left(\alpha \varphi_{\xi_{3}}^{(h)}+\alpha \varphi_{\xi_{4}}^{(h)}\right), \\
W_{F \times G}^{(4,7, h)}= & \int_{F \times G} d \underline{\xi} u_{N}^{(4,7, h)}(\xi) \frac{\sin \left(\alpha \varphi_{\xi_{1}}^{(h)}-\alpha \varphi_{\xi_{2}}^{(h)}\right)}{\left(\gamma^{h}\left|\xi_{1}-\xi_{2}\right|\right)^{1-\varepsilon}} \sin \left(\alpha \varphi_{\xi_{3}}^{(h)}+\alpha \varphi_{\xi_{4}}^{(h)}\right), \\
A_{J}^{(h)}= & \sum_{2}^{4} \sum_{i=1} \sum_{\varepsilon_{i}= \pm 1} \lambda_{h}^{n} \int_{J \times \ldots \times J} d \xi_{1} \ldots d \xi_{n} a_{N}^{\left(h, \varepsilon_{1}, \ldots, \varepsilon_{n}\right)}\left(\xi_{1}, \ldots, \xi_{n}\right) \\
& \cdot \cos \left(\alpha \sum_{1}^{n} \varepsilon_{i} \varphi_{\xi_{i}}^{(h)}\right), \\
C_{J}^{(h)}= & \sum_{2}^{4} \lambda_{h}^{n} \int_{J \times \ldots \times J} d \xi_{1} \ldots d \xi_{n} c_{N}^{(h)}\left(\xi_{1}, \ldots, \xi_{n}\right) .
\end{aligned}
$$

If $\varepsilon<2\left(1-\alpha^{2} / 8 \pi\right)$ (see Appendix A), there exist two positive constants $A$ and $\kappa$, independent of $N$, such that, for all $\Delta_{1}, \ldots, \Delta_{n}$ belonging to a pavement $Q_{h}$ of $R^{2}$ made of cubic tesserae with side size $\gamma^{-h}$ :

$$
\int_{\Delta_{1} \times \ldots \times \Delta_{n}} d \xi_{1} \ldots d \xi_{n}\left|u_{N}^{(n, i, h)}(\xi)\right| \leqq A \exp \left\{-\kappa \gamma^{h} d\left(\Delta_{1}, \ldots, \Delta_{n}\right)\right\}
$$

for $n=2,3,4$ and any $i\left(u_{N}^{(2,1, h)} \equiv u_{N}^{(2, h)}\right)$ and

$$
\begin{array}{r}
\int_{\Delta_{1} \times \ldots \times \Delta_{n}} d \xi_{1} \ldots d \xi_{n}\left|a_{N}^{\left(h, \varepsilon_{1}, \ldots, \varepsilon_{n}\right)}(\xi)\right| \leqq A \exp \left\{-\kappa \gamma^{h} d\left(\Delta_{1}, \ldots, \Delta_{n}\right)\right\}, \\
\int_{\Delta_{1} \times \ldots \times \Delta_{n}} d \xi_{1} \ldots d \xi_{n}\left|c_{N}^{(h)}(\xi)\right| \leqq A \exp \left\{-\kappa \gamma^{h} d\left(\Delta_{1}, \ldots, \Delta_{n}\right)\right\},
\end{array}
$$

where $d\left(\Delta_{1}, \ldots, \Delta_{n}\right)$ is the length of the shortest path connecting all the tesserae $\Delta_{1}, \ldots, \Delta_{n}$.

There is also a positivity property, which will be very important in the proof of the upper bound:

$$
u_{N}^{(2, h)}\left(\xi_{1}, \xi_{2}\right)>0
$$

Equation (3.17) will be used together with the following bounds:

$$
\begin{array}{cc}
\int_{J} d \xi_{3}\left|u^{(3, i, h)}(\xi)\right| \leqq A u_{N}^{(2, h)}\left(\xi_{1}, \xi_{2}\right) & i=1,2, \\
\int_{F} d \xi_{3} d \xi_{4}\left|u_{N}^{(4, i, h)}(\xi)\right| \leqq A u_{N}^{(2, h)}\left(\xi_{1}, \xi_{2}\right) & i \neq 2, \\
\int_{F} d \xi_{1} d \xi_{2}\left|u_{N}^{(4, i, h)}(\underline{\xi})\right| \leqq A u_{N}^{(2, h)}\left(\xi_{3}, \xi_{4}\right) & i=2,3,4,5,
\end{array}
$$


where $J \subset R$ and $F \subset R^{2}$ are arbitrary sets and $A$ is a suitable constant, independent of $N, h$. Let us now define:

$$
\begin{aligned}
\mathscr{D}_{h}= & \left\{\left(\xi_{1}, \xi_{2}\right) \in I \times I|| \sin \left(\frac{\alpha}{2} \varphi_{\xi_{1}}^{(h)}-\frac{\alpha}{2} \varphi_{\xi_{2}}^{(h)}\right) \mid \geqq B_{h}\left(\gamma^{h}\left|\xi_{1}-\xi_{2}\right|\right)^{1-\varepsilon},\right. \\
& \text { and } \left.B_{h}\left(\gamma^{h}\left|\xi_{1}-\xi_{2}\right|\right)^{1-\varepsilon} \leqq \delta\right\}
\end{aligned}
$$

where

$$
B_{h}=B\left(1+h^{3}\right) \log \left(e+\lambda^{-1}\right) .
$$

$B$ and $\delta<1$ will be chosen later. $J \subset I$ :

The definition of $\mathscr{D}_{h}$ is suggested by the following considerations. If we define, for

$$
\begin{aligned}
\hat{V}_{J}^{(h)}= & V_{0, J}^{(h)}-\lambda_{h}^{2} W_{J^{2} \backslash \mathscr{D}_{h}}^{(2, h)}+\lambda_{h}^{3} \sum_{1}^{2} W_{J^{2} \backslash \mathscr{D}_{h} \times J}^{(3, i, h)}+\lambda_{h}^{4} \sum_{3}^{5} W_{J^{2} \backslash \mathscr{D}_{h} \times J^{2} \backslash \mathscr{D}_{h}}^{(4, i, h)} \\
& +\lambda_{h}^{4} W_{J^{2}\left(\mathscr{D}_{h} \times J^{2}\right.}^{(4,1, h)}+\lambda_{h}^{4} W_{J^{2} \times J^{2} \backslash \mathscr{D}_{h}}^{(4,2, h)}+\lambda_{h}^{4} W_{J^{2} \backslash \mathscr{D}_{h} \times J^{2}}^{(4,6, h)} \\
& +\lambda_{h}^{4} W_{J^{2} \backslash \mathscr{D}_{h} \times J^{2}}^{(4,7, h)}+A_{J}^{(h)}+C_{J}^{(h)},
\end{aligned}
$$

then from Eqs. (3.2)-(3.16) it immediately follows that

$$
\left|\hat{V}_{J}^{(h)}\right| \leqq\left(\lambda_{h}+\lambda_{h}^{4}\right) B_{h}^{2} \bar{A}\left(\gamma^{2 h}|J|\right)
$$

for some constant $A$ independent of $h$ and $N$.

After understanding Appendix A the reader will observe that the bound (3.24) would be true also if we defined

$$
\begin{aligned}
\hat{V}_{J}^{(h)}= & V_{0, J}^{(h)}-\lambda_{h}^{2} W_{J^{2} \backslash \mathscr{D}_{h}}^{(2, h)}+\lambda_{h}^{3} \sum_{1}^{2} W_{J^{2} \times J}^{(3, i, h)}+\lambda_{h}^{4} \sum_{i \in\{1,4\}} W_{J^{2} \backslash \mathscr{D}_{h} \times J^{2}}^{(4, i, h)} \\
& +\lambda_{h}^{4} W_{J^{2} \backslash \mathscr{D}_{h} \times J^{2} \backslash \mathscr{D}_{h}}^{(4,3, h)}+\lambda_{h}^{4} \sum_{i \in\{6,7\}} W_{J^{2} \times J^{2}}^{(4, i, h)} .
\end{aligned}
$$

However the iterative procedure that we shall use in the proof of the upper bound of Eq. (2.17) suggests the definition (3.23).

The proof of Theorem 1 will be based on the estimate of $\int d \tilde{P}_{h} \exp \left(\hat{V}_{I}^{(h)}\right)$. However $\hat{V}_{I}^{(h)}$ is not a simple function of $\tilde{\varphi}^{(h)}$ as it depends on $\tilde{\varphi}^{(h)}$ through the set $\mathscr{D}_{h}$. Therefore, following the ideas developed in [2], we introduce another function which, as a function of $\tilde{\varphi}^{(h)}$, has essentially the same structure as $\tilde{V}_{J}^{(h)}$, thought of as a function of $\varphi^{(h)}$. We put, for $J \subset I$

$$
\begin{aligned}
H_{J}^{(h)}= & V_{0, J}^{(h)}-\lambda_{h}^{2} W_{J^{2} \backslash \mathscr{D}_{h-1}}^{(2, h)}+\lambda_{h}^{3} \sum_{1}^{2} W_{J^{2} \backslash \mathscr{Q}_{h-1} \times J}^{(3, i, h)}+\lambda_{h}^{4} \sum_{3}^{5} W_{J^{2} \backslash \mathscr{D}_{h-1} \times J^{2} \backslash \mathscr{D}_{h-1}}^{(4, i, h)} \\
& +\lambda_{h}^{4} W_{J^{2} \mid \mathscr{D}_{h-1} \times J^{2}}^{(4, h)}+\lambda_{h}^{4} W_{J^{2} \times J^{2} \backslash \mathscr{D}_{h-1}}^{(4,2, h)}+\lambda_{h}^{4} \sum_{6}^{7}{ }_{i} W_{J^{2} \backslash \mathscr{D}_{h-1} \times J^{2}}^{(4, i, h)}+A_{J}^{(h)}+C_{J}^{(h)}
\end{aligned}
$$


It is possible to see that $H_{J}^{(h)}$, thought of as a function of $\tilde{\varphi}^{(h)}$ at fixed $\varphi^{(h-1)}$, has the following structure:

$$
\begin{aligned}
H_{J}^{(h)}= & \sum_{1}^{4} n \sum_{\substack{0 \leqq p_{i} \leqq 2 \\
0 \leqq q_{i}}} \int_{J^{n}} d \xi_{1} \ldots d \xi_{n} v_{0}^{(n, \underline{p}, \underline{q})}(\underline{\xi})\left(\cos \frac{\alpha}{2} \tilde{\varphi}_{\xi_{1}}^{(h)}\right)^{p_{1}} \ldots\left(\sin \frac{\alpha}{2} \tilde{\varphi}_{\xi_{n}}^{(h)}\right)^{q_{n}} \\
& +\sum_{2}^{4} \sum_{1}^{2} \sum_{\substack{0 \leqq p_{i} \leqq 2 \\
0 \leqq q_{i} \leqq 2}} \int_{J^{n}} d \xi_{1} \ldots d \xi_{n} v_{1}^{(n, m, \underline{p}, \underline{q})}(\underline{\xi})\left[\frac{\sin \left(\frac{\alpha}{2} \tilde{\varphi}_{\xi_{1}}^{(h)}-\frac{\alpha}{2} \tilde{\varphi}_{\xi_{2}}^{(h)}\right)}{\left(\gamma^{h}\left|\xi_{1}-\xi_{2}\right|\right)^{1-\varepsilon}}\right] m \\
& \cdot\left(\cos \frac{\alpha}{2} \tilde{\varphi}_{\xi_{1}}^{(h)}\right)^{p_{1}} \ldots\left(\sin \frac{\alpha}{2} \tilde{\varphi}_{\xi_{n}}^{(h)}\right)^{q_{n}} \\
& +\int_{J^{4}} d \xi_{1} \ldots d \xi_{4} v_{2}(\xi) \frac{\sin \left(\frac{\alpha}{2} \tilde{\varphi}_{\xi_{1}}^{(h)}-\frac{\alpha}{2} \tilde{\varphi}_{\xi_{2}}^{(h)}\right)}{\left(\gamma^{h}\left|\xi_{1}-\xi_{2}\right|\right)^{1-\varepsilon}} \frac{\sin \left(\frac{\alpha}{2} \tilde{\varphi}_{\xi_{3}}^{(h)}-\frac{\alpha}{2} \tilde{\varphi}_{\xi_{4}}^{(h)}\right)}{\left(\gamma^{h}\left|\xi_{3}-\xi_{4}\right|\right)^{1-\varepsilon}}
\end{aligned}
$$

with the functions $v_{i}(\xi)$ satisfying the following estimate, for all $\Delta_{1}, \ldots, \Delta_{n} \in Q_{h}$ :

$$
\int_{\Delta_{1} \times \ldots \times \Delta_{n}} d \xi_{1} \ldots d \xi_{n}\left|v_{i}(\xi)\right| \leqq \bar{H}_{h} \exp \left\{-\kappa \gamma^{h} d\left(\Delta_{1}, \ldots, \Delta_{n}\right)\right\},
$$

where, for a suitable constant $\bar{H}$ :

$$
\bar{H}_{h}=\left(\lambda_{h}+\lambda_{h}^{4}\right) \bar{H} B_{h-1}^{2} .
$$

Let us now consider the $\tilde{P}_{h}$-measurable event

$$
E_{\Delta}^{b}=\left\{\tilde{\varphi}^{(h)}\left|\sup _{\xi \in \Delta}\right| \tilde{\varphi}_{\xi}^{(h)} \mid+\sup _{\xi_{1}, \xi_{2} \in \Delta} \frac{\left|\tilde{\varphi}_{\xi_{1}}^{(h)}-\tilde{\varphi}_{\xi_{2}}^{(h)}\right|}{\left(\gamma^{h}\left|\xi_{1}-\xi_{2}\right|\right)^{1-\varepsilon}} \leqq b\left(1+\gamma^{h} d(\Delta, I)\right)\right\},
$$

where $\Delta \in Q_{h}$ and $b>0$. We shall denote by $\chi_{\Delta}^{b}$ the characteristic function of $E_{\Delta}^{b}$ and by $\dot{\chi}_{\Delta}^{b}=1-\chi_{\Delta}^{b}$ the characteristic function of its complement. If $\mathscr{R}=\left(\Delta_{1}, \Delta_{2}, \ldots\right)$ denotes a subsets of $Q_{h}$, which will be identified with $\bigcup_{\Delta \in \mathscr{R}} \Delta, \mathscr{R}^{\mathbf{c}}$ will be its complement and we shall define :

$$
\begin{aligned}
\dot{\chi}_{\mathscr{R}}^{b} & =\prod_{\Delta \in R} \dot{\chi}_{\Delta}^{b}, \quad \chi_{\mathscr{R}^{c}}^{b}=\prod_{\Delta \in \mathscr{R}^{c}} \chi_{\Delta}^{b}, \\
\hat{\mathscr{R}} & =\left\{\Delta \in Q_{h} \mid \gamma^{h} d(\Delta, \mathscr{R}) \leqq b^{3}\right\} .
\end{aligned}
$$

$H_{J}^{(h)}$ has an important property, which is expressed by the following lemma:

Lemma 1. Let $\gamma$ be fixed close enough to 1 and $0<\varepsilon<1$. For any integer $t \geqq 0$ there exist constants $b^{*}, G, \varrho, \varrho^{\prime}$ depending only on $\varepsilon, t$, and $\kappa$ such that, if $b>b^{*}$ and $\mathscr{R}$ is $a$ subset of $Q_{h}$

$$
\begin{aligned}
& \int d \tilde{P}_{h} \dot{\chi}_{\mathscr{R}}^{b} \chi_{\mathscr{R}}^{b} \exp \left(H_{J \backslash \hat{\mathscr{R}}}^{(h)} \leqq \exp \left\{\delta\left(b, \bar{H}_{h}\right) \gamma^{2 h}|I|+\delta^{\prime}\left(b, \bar{H}_{h}\right) \gamma^{2 h}|\hat{\mathscr{R}} \cap J|\right\}\right. \\
& \cdot \exp \left\{\sum_{1}^{t} \frac{\tilde{E}_{h}\left(H_{J}^{(h)}, k\right)}{k !}\right\}\left(\int d \tilde{P}_{h} \dot{\chi}_{\mathscr{R}}^{b}\right)^{1 / 2},
\end{aligned}
$$


where

$$
\begin{aligned}
\delta\left(b, \bar{H}_{h}\right) & =G\left[\left(\bar{H}_{h} b^{\varrho} e^{\varrho \bar{H}_{h} b^{\varrho}}\right)^{t+1}+e^{-\varrho^{\prime} b^{2}+\varrho \bar{H}_{h} b^{\varrho}}\right] \\
\delta^{\prime}\left(b, \bar{H}_{h}\right) & =G \bar{H}_{h} b^{\varrho} .
\end{aligned}
$$

Furthermore, if $b>b^{*}$

$$
\begin{aligned}
\int d \tilde{P}_{h} \chi_{Q_{h}}^{b} \exp \left(H_{J}^{(h)}\right) \geqq & \exp \left\{-\gamma^{2 h}|I| \delta\left(b, \bar{H}_{h}\right)\right\} \\
& \cdot \exp \left\{\sum_{1}^{t} \frac{\tilde{E}_{h}^{T}\left(H_{J}^{(h)} ; k\right)}{k !}\right\} .
\end{aligned}
$$

Thanks to the scaling property (2.4) of the fields $\tilde{\varphi}^{(h)}$, it is sufficient to prove this lemma in the case $h=0$. The proof can be done exactly as the proof of Lemma 1 in [2], using the support properties of $\tilde{\varphi}^{(0)}$ proven in [3] and some stronger results discussed in Appendix $\mathrm{C}$, where the proof is sketched. In Appendix $\mathrm{C}$ the following proposition, needed later, is also proven.

Proposition 1. $\forall \varepsilon>0$ there exist $\bar{b}, e_{1}, e_{2}$ such that, if $b \geqq \bar{b}$

$$
\int d \tilde{P}_{0} \dot{\chi}_{\mathscr{R}}^{b} \leqq \prod_{\Delta \in \mathscr{R}} \exp \left\{e_{1}-e_{2} b^{2}(1+d(\Delta, I))\right\}
$$

for any $\mathscr{R} \subset Q_{1}$.

\section{The Lover Bound}

The lower bound in Eq. (2.17) is obtained by recursively applying Lemma 1. We start from the inequality:

$$
\int\left[\prod_{h=0}^{N} \chi_{h}^{B}\left(\varphi^{(h)}\right)\right] \exp \tilde{V}_{I}^{(N)} \prod_{h=0}^{N} d \tilde{P}_{h} \leqq \int \exp V_{I}^{(N)} d P_{N},
$$

where $\chi_{h}^{B}\left(\varphi^{(h)}\right)$ is the characteristic function of the event:

$$
\left\{\varphi^{(h)}\left|\sup _{\xi \in \Delta}\right| \varphi_{\xi}^{(h)} \mid+\sup _{\xi_{1}, \xi_{2} \in \Delta} \frac{\left|\varphi_{\xi_{1}}^{(h)}-\varphi_{\xi_{2}}^{(h)}\right|}{\left(\gamma^{h}\left|\xi_{1}-\xi_{2}\right|\right)^{1-\varepsilon}} \leqq B_{h}\left(1+\gamma^{h} d(\Delta, I)\right), \quad \forall \Delta \in Q_{h}\right\},
$$

and $B_{h}$ is defined as in Eq. (3.22). From Eqs. (2.2), (3.30), (3.31), and (4.2) it follows that

$$
\chi_{h-1}^{B}\left(\varphi^{(h-1)}\right) \chi_{h}^{B}\left(\varphi^{(h)}\right) \geqq \chi_{h-1}^{B}\left(\varphi^{(h-1)}\right) \chi_{Q_{h}}^{b_{h}}, \quad h \geqq 1,
$$

where

$$
b_{h}=\frac{B}{2}\left(1+h^{2}\right) \log \left(e+\lambda^{-1}\right)
$$

Then we have, by Eqs. (3.26), (3.34), and (4.3), if $\frac{B}{2}>b^{*}$ :

$$
\begin{aligned}
& \int \prod_{h=0}^{k} \chi_{h}^{B}\left(\varphi^{(h)}\right)\left(\exp \tilde{V}_{I}^{(k)}\right) \prod_{h=0}^{k} d \tilde{P}_{h} \geqq \int \prod_{h=0}^{k-1} \chi_{h}^{B}\left(\varphi^{(h)}\right) \int d \tilde{P}_{k} \chi_{Q_{\mathbf{k}}}^{b_{\mathbf{k}}}\left(\exp \tilde{V}_{I}^{(k)}\right) \\
& \geqq \exp \left\{-\gamma^{2 k} \delta\left(b_{k}, \bar{H}_{k}\right)|I|\right\} \int \exp \left\{\sum_{1}^{4} \frac{1}{n} n ! \tilde{\mathscr{E}}_{k}^{T}\left(\tilde{V}_{I}^{(k)} ; n\right)\right\} \prod_{h=0}^{k-1} \chi_{h}^{B}\left(\varphi^{(h)}\right) d \tilde{P}_{k-1},
\end{aligned}
$$


and $\delta\left(b, \bar{H}_{k}\right)$ is defined as in Eq. (3.33) with $t=4$. Furthermore, by noticing that in Eq. (3.28) the real bound is of order $\lambda_{h}^{n}$ for terms of order $\lambda^{n}$ (see Appendix A), it is easy to see that $[\text { see Eq. (2.9) }]^{4}$ :

$$
\chi_{k-1}^{B}\left(\varphi^{(k-1)}\right)\left|\tilde{V}_{I}^{(k-1)}-\sum_{1}^{4} \frac{1}{n !} \tilde{\mathscr{E}}_{k}^{T}\left(\tilde{V}_{I}^{(k)}, n\right)\right| \leqq \gamma^{2 k} \delta\left(b_{k}, \bar{H}_{k}\right)|I| .
$$

Finally from the definition (2.9) it immediately follows that

$$
\tilde{V}_{I}^{(-1)}=\mathscr{E}_{N}\left(V_{0, I}^{(N)}\right)+\frac{1}{3 !} \mathscr{E}_{N}^{T_{N}}\left(V_{0, I}^{(N)} ; 3\right)
$$

The lower bound in Eq. (2.17) follows Eqs. (4.6) and (4.7), putting

$$
E_{-}(\lambda)=\sum_{0}^{\infty} \gamma^{2 k} \delta\left(b_{k}, \bar{H}_{k}\right) \text {. }
$$

By Eqs. (3.29) and (3.33) $E_{-}(\lambda)$ is finite for all $\alpha^{2}<\frac{32}{5} \pi$ and satisfy Eq. (2.19). Then, as regards the lower bound, there is no necessity of restricting $\alpha^{2}$ to the values less than $\bar{\alpha}^{2}<\frac{32}{5} \pi$. Furthermore, by increasing the value of $t$ in Eq. (2.9) and doing the algebra needed in order to prove the bound (3.28), it is also possible to obtain the lower bound for all values of $\alpha^{2}<8 \pi$. The proof of the lower bound can be extended to $Z_{I}(f)$ [see Eq. (2.16)] without any real difference, since $\exp \varphi^{(N)}(f)$ $=\prod_{h=0}^{N} \exp \tilde{\varphi}^{(h)}(f)$ and

$$
\tilde{\varphi}^{(h)}(f)=\sum_{\Delta \in Q_{h}} \gamma^{-2 h} \int_{\gamma^{h_{\Delta}}} z_{\xi}^{(h)} f\left(\gamma^{-h} \xi\right) d \xi
$$

Proceeding as before, it is easy to see that

$$
\begin{aligned}
& \int \prod_{h=0}^{k} \chi_{h}^{B}\left(\varphi^{(h)}\right) \exp \left(\tilde{V}_{I}^{(k)}+\varphi^{(k)}(f)\right) d P_{k} \geqq \int \prod_{h=0}^{k-1} \chi_{h}^{B}\left(\varphi^{(h)}\right) \exp \left(\tilde{V}_{I}^{(k-1)}+\varphi^{(k-1)}(f) d P_{k-1}\right. \\
& \quad \cdot \exp \left\{-\gamma^{2 k} \delta\left(b_{k} \bar{H}_{k}\right)|I|-G\left(\bar{H}_{k}+\gamma^{-2 k}\right) b_{k}^{\varrho}|\operatorname{supp} f| \cdot\|f\|_{\infty}\right\} .
\end{aligned}
$$

which solves the problem.

\section{The Upper Bound}

Also the proof of the upper bound in Eq. (2.17) is reduced to Lemma 1. Now, however, there is a problem which does not appear in the lower bound. In fact, in order to prove that is possible to neglect in the "effective interaction" $\tilde{V}_{J}^{(h)}$ the regions $\mathscr{D}_{h}$, where the field is rough, we need some not obvious positivity properties. An analogous problem was present in $\varphi_{3}^{4}$ theory (see [2]), where the solution was much simpler. In this case we could extend the method used in [2]

4 In the following $\delta\left(b, \bar{H}_{k}\right)$ and $\delta^{\prime}\left(b, \bar{H}_{k}\right)$ will denote the expressions (3.33) with a suitable value of $G$, in general larger than in Lemma 1 
until $\alpha^{2}=\bar{\alpha}^{2}$, which is a not trivial result, since $\bar{\alpha}^{2}>6 \pi$, the threshold for the appearance of four-particle clusters.

Let us define:

$$
\begin{aligned}
& \mathscr{R}_{h}\left(\tilde{\varphi}^{(h)}\right)=\left\{\Delta \in Q_{h} \mid \exists \xi, \eta \in \Delta \text { such that }\left|\sin \frac{\alpha}{2}\left(\tilde{\varphi}_{\xi}^{(h)}-\tilde{\varphi}_{\eta}^{(h)}\right)\right|\right. \\
& \geqq \bar{B}_{h}\left(\gamma^{h}|\xi-\eta|\right)^{1-\varepsilon}\left(1+\gamma^{h} d(\Delta, I)\right) \text { or } \exists \xi \in \Delta \text { such that }\left|\tilde{\varphi}_{\xi}^{(h)}\right| \\
& \left.\geqq \bar{B}_{h}\left(1+\gamma^{h} d(\Delta, I)\right)\right\},
\end{aligned}
$$

where

$$
\bar{B}_{h}=\bar{B}\left(1+h^{2}\right) \log \left(e+\lambda^{-1}\right)
$$

and $\bar{B}$ will be chosen later.

If we define $\hat{V}_{J}^{(h)}$ and $H_{J}^{(h)}$ as in Sect. 3, we can summarize the properties of the effective interaction that we need, in the following Lemma:

Lemma 2. If $\alpha^{2}<\bar{\alpha}^{2}$, there exist positive constants $\underline{C}, \varrho, \delta_{0}, \sigma, \bar{B}_{0}$ and an integer valued function $\bar{h}(\bar{B}, \lambda)$ such that, if $h \geqq \bar{h}(\bar{B}, \lambda), \delta \leqq \delta_{0}, \bar{B} \geqq \bar{B}_{0}$ and $B=\sigma \bar{B}$ ( $\delta$ and $B$ are the constants entering in the definition (3.21) of $\left.\mathscr{D}_{h}\right), \forall J \subset I$ :

i)

$$
\begin{gathered}
\tilde{V}_{J}^{(N-1)} \leqq \hat{V}_{J}^{(N-1)}, \\
\hat{V}_{J}^{(h)} \leqq \bar{A} \lambda_{h} B_{h}^{2} \gamma^{2 h}\left|\hat{\mathscr{R}}_{h} \cap J\right|+H_{J \backslash \hat{\mathscr{R}}_{h}}^{(h)},
\end{gathered}
$$

where $\hat{\mathscr{R}}_{h}=\left\{\Delta \in Q_{h} \mid \gamma^{h} d\left(\Delta, \mathscr{R}_{h}\left(\varphi^{(h)}\right)\right) \leqq \bar{B}_{h}^{3}\right\}$ is identified with $\bigcup_{\Delta \in \mathscr{R}_{h}} \Delta$,

$$
\text { iii) } \quad \sum_{1}^{k} \frac{1}{n !} \tilde{\mathscr{E}}_{h}^{T}\left(H_{J}^{(h)} ; n\right)-\hat{V}_{J}^{(h-1)} \leqq\left[\gamma^{2 h} \delta\left(\bar{B}_{h}, \bar{H}_{h}\right)+c \lambda^{4+\tau} \gamma^{-\varrho h}\right]|I|
$$

with $\tau<\frac{4 \pi}{\alpha^{2}}-\frac{1}{2}$ $\bar{B})$.

iv) $\bar{h}(\bar{B}, \lambda)=0$ if $\lambda$ is small enough (but this property does not hold uniformly in

Proof. Equation (5.3) is an immediate consequence of Eqs. (3.17)-(3.20) and the fact that for $h$ large enough, the second order term $-\lambda_{h}^{2} W_{J}^{(2, h)}$ dominates the other ones. In order to prove the bound (5.4), observe that it is possible to choose $\delta_{0}<1$ and $\sigma>1$ so that, if $\delta \leqq \delta_{0}$ and $B=\sigma \bar{B}$ :

a) $\quad(\xi, \eta) \in \mathscr{D}_{h-1} \cap\left(I \backslash \mathscr{R}_{h}\right)^{2} \Rightarrow\left|\sin \frac{\alpha}{2}\left(\varphi_{\xi}^{(h)}-\varphi_{\eta}^{(h)}\right)\right| \geqq \tilde{B}_{h}^{\prime}\left(\gamma^{h}|\xi-\eta|\right)^{1-\varepsilon}$

with

$$
\tilde{B}_{h}=\tilde{B}\left(1+h^{3}\right) \log \left(e+\lambda^{-1}\right), \quad \tilde{B}>0,
$$

b)

$$
\mathscr{D}_{h} \backslash \mathscr{D}_{h-1} \subset \hat{\mathscr{R}}_{h} \times \hat{\mathscr{R}}_{h} \text {. }
$$

Equations (5.6) and (5.8) are immediate consequences of the definitions (3.21) and (5.1). 
Using Eq. (3.24), we can write:

$$
\hat{V}_{J}^{(h)} \leqq \bar{A} \lambda_{h} B_{h}^{2} \gamma^{2 h}\left|\hat{\mathscr{R}}_{h} \cap J\right|+\hat{V}_{J \backslash \hat{\mathscr{R}}_{h}}^{(h)} .
$$

Furthermore, by Eq. (5.6) and Eqs. (3.17)-(3.20), we can prove that there exist $\bar{h}(\bar{B}, \lambda)$, such that, if $h \geqq \bar{h}(\bar{B}, \lambda)$

$$
\hat{V}_{J \backslash \hat{\mathscr{R}}_{h}}^{(h)}=\hat{V}_{J \backslash \hat{\mathscr{R}}_{h}}^{(h)}\left(\mathscr{D}_{h}\right) \leqq \hat{V}_{J \backslash \hat{\mathscr{R}}_{h}}^{(h)}\left(\mathscr{D}_{h} \cup \mathscr{D}_{h-1}\right) .
$$

But Eq. (5.8) and the definition of $H_{J}^{(h)}$ imply that

$$
\hat{V}_{J \backslash \hat{\mathscr{R}}_{h}}^{(h)}\left(\mathscr{D}_{h} \cup \mathscr{D}_{h-1}\right)=H_{J \backslash \hat{\mathscr{R}}_{h}}^{(h)} .
$$

Equations (5.9)-(5.11) imply Eq.(5.4).

In order to prove Eq. (5.5) we first observe that, by the same argument used in the lower bound [see Eq. (4.6)]:

$$
\begin{aligned}
& \sum_{1}^{4} \frac{1}{n !} \tilde{\mathscr{E}}_{h}^{T}\left(H_{J}^{(h)}, n\right)-\left[\sum_{1}^{4} \frac{1}{n !} \tilde{\mathscr{E}}_{h}^{T}\left(H_{J}^{(h)}, n\right)\right]_{4} \\
& \leqq \gamma^{2 h} \delta\left(\bar{B}_{h}, \bar{H}_{h}\right)|I| .
\end{aligned}
$$

Then it is sufficient to show that there exists $\bar{B}_{0}$ such that

$$
\left[\sum_{1}^{k} n \frac{1}{n !} \tilde{\mathscr{E}}_{h}^{T}\left(H_{J}^{(h)} ; n\right)\right]_{4}-\hat{V}_{J}^{(h-1)} \leqq c \lambda^{4} \gamma^{-\varrho h}|I|, \quad \bar{B} \geqq \bar{B}_{0} .
$$

The bound (5.13) is at the origin of the restriction (2.14) on $\alpha^{2}$. In fact $\varrho \rightarrow 0$ if $\alpha^{2} \rightarrow \bar{\alpha}^{2}$, so that the "error" in the right hand side of (5.13) is not summable in $h$ any more. Equation (5.13), depends on the detailed structure of $\hat{V}_{J}^{(h)}$ and will be proven in Appendix B.

We can now prove the upper bound on the partition function. First observe that the integration of $\varphi^{(N)}$ can be easily done, since $V_{I}^{(N)}=H_{I}^{(N)}$ is bounded. By Eqs. (3.32) and (5.12), we have (see footnote 4):

$$
\begin{aligned}
& \int d \tilde{P}_{N} \exp \left(V_{I}^{(N)}\right)=\sum_{\mathscr{R} C Q_{N}} \int d \tilde{P}_{N} \dot{\chi}_{\mathscr{R}_{N}}^{\bar{B}_{N}} \chi_{\mathscr{R}_{N}}^{\bar{B}_{c}} \exp \left(H_{I}^{(N)}\right) \\
& \leqq \sum_{\mathscr{R} \subset Q_{N}} \int d \tilde{P}_{N} \dot{\chi}_{\mathscr{R}_{N}} \bar{B}_{\mathscr{R}_{N}} \chi_{\mathscr{B}_{\mathscr{R}_{N}}} \exp \left(H_{I \backslash \hat{\mathscr{R}}_{N}}^{(N)}\right) \exp \left(\lambda_{N} \bar{A} \gamma^{2 N}\left|I \cap \hat{\mathscr{R}}_{N}\right|\right) \\
& \leqq \sum_{\mathscr{R} \subset Q_{N}} \exp \left(\delta\left(\bar{B}_{N}, \bar{H}_{N}\right) \gamma^{2 N}|I|+\delta^{\prime}\left(\bar{B}_{N}, \bar{H}_{N}\right) \gamma^{2 N}\left|I \cap \hat{\mathscr{R}}_{N}\right|\right) \exp \left(\tilde{V}_{I}^{(N-1)}\right) \\
& \quad \cdot\left(\int d \tilde{P}_{N} \dot{\chi}_{\mathscr{R}_{N}}\right)^{1 / 2} .
\end{aligned}
$$

The sum in (5.14) can be easily estimated using Eq. (3.27), which gives

$$
\begin{aligned}
& \sum_{\mathscr{R} \subset Q_{N}} \exp \left(\delta^{\prime}\left(\bar{B}_{N}, \bar{H}_{N}\right) \gamma^{2 N}\left|I \cap \hat{\mathscr{R}}_{N}\right|\right)\left(\int d \tilde{P}_{N} \dot{\chi}_{\mathscr{B}_{N}}^{\bar{B}_{N}}\right)^{1 / 2} \\
& \quad \leqq \prod_{\Delta \in Q_{N}}\left[1+\exp \left(d^{\prime}\left(\bar{B}_{N}, \bar{H}_{N}\right)+\frac{c_{1}}{2}-\frac{c_{2}}{2} \bar{B}_{N}^{2}\left(1+\gamma^{N} d(\Delta, I)\right)\right)\right] \\
& \quad \leqq \exp \left[c_{3} e^{\delta^{\prime}\left(\bar{B}_{N}, \bar{H}_{N}\right)+\frac{c_{1}}{2}-\frac{c_{2}}{2} \bar{B}_{N}^{2}}\right] \gamma^{2 N}|I|
\end{aligned}
$$


The effect of this sum is then simply to modify the constant $G$ in the definition of $\delta\left(B_{N}, \bar{H}_{N}\right)$ and we can write, using also Eq. (5.3)

$$
\int d \tilde{P}_{N} \exp \left(V_{I}^{(N)}\right) \leqq \exp \left(V_{I}^{(N-1)}\right) \exp \left[\delta\left(\bar{B}_{N}, \bar{H}_{N}\right) \gamma^{2 N}|I|\right]
$$

In the same way, using Eqs. (5.4) and (5.5), we can prove that, if $h \geqq \bar{h}(\bar{B}, \lambda)$ :

$$
\int d \tilde{P}_{h} \exp \left(\hat{V}_{I}^{(h)}\right) \leqq \exp \left(\hat{V}_{I}^{(h-1)}\right) \exp \left[\delta\left(\bar{B}_{h}, \bar{H}_{h}\right) \gamma^{2 h}|I|+c \lambda^{4} \gamma^{-\varrho h}|I|\right] .
$$

From Eqs. (5.16) and (5.17) the upper bound immediately follows with:

$$
E_{+}(\lambda)=\sum_{\bar{h}}^{\infty}\left[\delta\left(\bar{B}_{h}, \bar{H}_{h}\right) \gamma^{2 h}+c \lambda^{4} \gamma^{-\varrho h}\right]+\sup _{I} \sup _{\varphi^{(\bar{h}-1)}}\left(|I|^{-1} \hat{V}_{I}^{(\bar{h}-1)}\right)
$$

Equation (2.20) is at this point obvious. Also the extension to $Z_{I}(f)$ is easy.

Acknowledgements. We are indebted to R. Seiler for suggesting the problem and to J. Fröhlich, R. Seiler, and K. Schrader for useful discussions and comments.

\section{Appendix A}

In this appendix we shall give some details about the structure of $\tilde{V}_{I}^{(h)}$, defined by Eqs. (2.9) and (2.15). Given the $n$ "vertices" $\left\{\xi_{1}, \ldots, \xi_{n}\right\}$, we define

$$
\underline{\xi}=\left(\xi_{1}, \ldots, \xi_{n}\right), \quad \underline{\varphi}^{(h)}=\left(\varphi_{\xi_{1}}^{(h)}, \ldots, \varphi_{\xi_{n}}^{(h)}\right), \quad \underline{\varepsilon}=\left(\varepsilon_{1}, \ldots, \varepsilon_{n}\right),
$$

where $\varepsilon_{i}= \pm 1, i=1, \ldots, n$. Furthermore, if $X=\left\{i_{1}, \ldots, i_{r}\right\}$ is a subset of $\{1, \ldots, n\}$, we put

$$
\underline{\xi}_{X}=\left(\xi_{1}, \ldots, \xi_{i_{2}}\right), \quad \underline{\varphi}_{X}^{(h)}=\left(\varphi_{\xi_{1}}^{(h)}, \ldots, \varphi_{\xi_{i_{r}}}^{(h)}\right), \quad \underline{\varepsilon}_{X}=\left(\varepsilon_{i_{1}}, \ldots, \varepsilon_{i_{r}}\right) .
$$

If $\left\{X_{1}, \ldots, X_{s}\right\}$ is a partition of $\{1, \ldots, n\}$, we define:

$$
U_{X_{1}, \ldots, X_{s}}^{(h, k)}(\underline{\varepsilon})=\sum_{1 \leqq u<v \leqq s} \sum_{\substack{i \in X_{u} \\ j \in X_{v}}} \varepsilon_{i} \varepsilon_{j} \sum_{h}^{k} r \tilde{C}_{0}\left(\gamma^{r} \mid \xi_{i}-\xi_{j}\right) .
$$

In the sequel we shall use the following relations, which are simple consequences of the Wick product's definition:

$$
\begin{aligned}
& \tilde{\mathscr{E}}_{h}\left(: e^{i \alpha \underline{\mathbf{X}}_{\mathbf{X}} \underline{\varphi}_{\mathbf{X}}^{(h)}}:\right)=: e^{i \alpha \underline{\underline{\varepsilon}} \mathbf{X} \varphi_{\mathbf{X}}^{(h-1)}}: \\
& : e^{i \alpha \underline{\varepsilon} \mathbf{X} \Psi_{\mathbf{X}}^{(h)}}:=e^{\frac{\alpha^{2}}{2} \mathscr{E}_{h}\left(\left[\underline{\underline{E}} \mathbf{X} \underline{\varphi} \underline{\mathbf{X}}^{(h)}\right]^{2}\right)} e^{i \alpha \underline{\varepsilon} \mathbf{X} \varphi_{\mathbf{X}}^{(h)}} \\
& =\left(\gamma^{\frac{\alpha^{2}}{4 \pi} h}\right)^{r} e^{\alpha^{2} U_{{ }_{1}}^{(0, h)},{ }_{, r}(\varepsilon)} e^{i \alpha \underline{\Sigma}_{X} \underline{\varphi}_{X}^{(h)}}, \quad X=\left\{i_{1}, \ldots, i_{r}\right\} .
\end{aligned}
$$

We recall also a well-known property of the truncated expectation:

$$
\tilde{\mathscr{E}}_{h}\left(f_{1}, \ldots, f_{r}\right)=\sum_{D \in \mathscr{D}(1, \ldots, r)}(-1)^{|D|-1}(|D|-1) ! \prod_{\gamma \in D} \tilde{\mathscr{E}}_{h}\left(\prod_{i \in \gamma} f_{i}\right)
$$

where $\mathscr{D}(1, \ldots, r)$ is the set of partitions of $\{1, \ldots, r\}$ and $|D|$ denotes the number of elements in $D$. 
Using Eqs. (A.4)-(A.6), it is easy to show that, given a partition $\left\{X_{1}, \ldots, X_{s}\right\}$ of $\{1, \ldots, u\}$

$$
\frac{1}{s !} \tilde{\mathscr{E}}_{h} T\left(: e^{i \alpha \underline{\varepsilon} \mathbf{x}_{1} \varphi_{\mathbf{X}_{1}}^{(h)}}:, \ldots,: e^{i \alpha \underline{\mathbf{E}}_{\mathbf{X}_{s}} \varphi_{\mathbf{X}_{s}}^{(h)}}:\right)=F^{(h)}\left(X_{1}, \ldots, X_{s} ; \underline{\varepsilon}\right): e^{i \alpha \underline{\underline{\varepsilon}} \underline{\varphi}^{(h-1)}}:
$$

where

$$
\begin{aligned}
& F^{(h)}\left(X_{1}, \ldots, X_{s} ; \underline{\varepsilon}\right)=\frac{1}{s !} e^{-\alpha^{2} U_{X_{1}}^{(0, h-1), X_{s}}(\varepsilon)}\left[e^{-\alpha^{2} U_{X_{1}}^{(h, h)}, X_{s}(\varepsilon)}\right]_{T}, \\
& {\left[e^{-\alpha^{2} U_{\left.X_{1} \cdot h\right)}^{(h, h)} \cdot X_{s}(\underline{g})}\right]_{T}=\sum_{D \in \mathscr{D}(1, \ldots, s)}(-1)^{|D|-1}(|D|-1) ! \prod_{\substack{\gamma \in D \\
\gamma=\left\{i_{1}, \ldots, i_{\hat{r}}\right\}}} \mathrm{e}^{-\alpha^{2} U_{X_{i 1}}^{(h h)} \cdot x_{i} \hat{r} .}}
\end{aligned}
$$

Equation (A.7) allows us to obtain very easily the expression of $\tilde{V}_{I}^{(h)}$ for any $t$. For simplicity we shall give the result for $t=4$ :

$$
\begin{aligned}
\tilde{V}_{I}^{(h)}= & V_{0, I}^{(h)}+\sum_{h+1}^{N} \sum_{2}^{4} \sum_{1}^{m_{u}} \lambda_{i_{u}} \lambda^{u} \sum_{\varepsilon_{1}, \ldots, \varepsilon_{u}= \pm 1} \int_{I \times \ldots \times I} d \xi_{1} \ldots d \xi_{u} G_{u, i_{u}}^{(i)}(\underline{\xi}, \underline{\varepsilon}) \\
& \cdot\left[: e^{i \alpha \underline{\varepsilon} \cdot \underline{\varphi}^{(h)}}:-\frac{1+(-1)^{n}}{2}\right] \\
& -\sum_{0}^{h} \sum_{2}^{4} \sum_{u}^{m_{u}} \sum_{1}^{m_{i}} \lambda^{u} \frac{1+(-1)^{u}}{2} \sum_{\varepsilon_{1}, \ldots, \varepsilon_{u}= \pm 1} \int_{I \times \ldots \times I} d \xi_{1} \ldots d \xi_{u} G_{u, i_{u}}^{(i)}(\underline{\xi}, \underline{\varepsilon}),
\end{aligned}
$$

where $m_{2}=1, m_{3}=2, m_{4}=5$, and

$$
\begin{aligned}
& G_{2,1}^{(i)}(\underline{\xi}, \underline{\varepsilon})=F^{(i)}(1,2 ; \underline{\varepsilon}), \\
& G_{3,1}^{(i)}(\underline{\xi}, \underline{\varepsilon})=F^{(i)}(1,2,3 ; \underline{\varepsilon}), \\
& G_{3,2}^{(i)}(\underline{\xi}, \underline{\varepsilon})=2 F^{(i)}((1,2), 3 ; \underline{\varepsilon}) \sum_{i+1}^{N} F^{(J)}\left(1,2 ; \underline{\varepsilon}_{(1,2)}\right), \\
& G_{4,1}^{(i)}(\underline{\xi}, \underline{\varepsilon})=F^{(i)}(1,2,3,4 ; \underline{\varepsilon}) \\
& G_{4,2}^{(i)}(\underline{\xi}, \underline{\varepsilon})=2 F^{(i)}((1,2,3), 4 ; \underline{\varepsilon}) \sum_{i+1}^{N} F^{(J)}\left(1,2,3 ; \underline{\varepsilon}_{(1,2,3)}\right) \\
& G_{4,3}^{(i)}(\underline{\xi}, \underline{\varepsilon})=F^{(i)}((1,2),(3,4) ; \underline{\varepsilon}) \sum_{i+1}^{N} F^{(J 1)}\left(1,2 ; \underline{\varepsilon}_{(1,2)}\right) \sum_{i+1}^{N} J_{J_{2}} F^{\left(J_{2}\right)}\left(3,4 ; \underline{\varepsilon}_{(3,4)}\right), \\
& G_{44}^{(i)}(\underline{\xi}, \underline{\varepsilon})=3 F^{(i)}((1,2), 3,4 ; \underline{\varepsilon}) \sum_{i+1}^{N} F^{(J)}\left(1,2 ; \underline{\varepsilon}_{(1,2)}\right), \\
& G_{45}^{(i)}(\underline{\xi}, \underline{\varepsilon})=4 F^{(i)}((1,2,3), 4 ; \underline{\varepsilon}) \sum_{i+1}^{N} F^{(J)}\left((1,2), 3 ; \underline{\varepsilon}_{(1,2,3)}\right) \sum_{J+1}^{N} F^{(J)}\left(1,2 ; \underline{\varepsilon}_{(1,2)}\right) .
\end{aligned}
$$

In Eqs. (A.13) and (A.15)-(A.18) is understood that the left hand side is zero if the right hand side is meaningless. The expression (3.1) of $\tilde{V}_{I}^{(h)}$ is obtained by singling out the terms depending on $\varphi^{(h)}$ of "total charge" $Q=\sum_{i}^{n} \varepsilon_{i}=0$ and the terms 
containing the function $F^{(J)}\left(r, s ; \underline{\varepsilon}_{(r, s)}\right)$ with $\varepsilon_{r}+\varepsilon_{s}=0$, which give rise to the $W^{(u, i, h)}$ terms. The other terms are collected in $A_{I}^{(h)}$ and $C_{I}^{(h)}$. We have:

$$
\begin{aligned}
& \lambda_{h}^{2} u_{N}^{(2, h)}\left(\xi_{1}, \xi_{2}\right)=\left(\gamma^{h}\left|\xi_{1}-\xi_{2}\right|\right)^{2(1-\varepsilon)} 2 \lambda^{2} \sum_{h+1}^{N} G_{2,1}^{(i)}(\xi ;(+,-)) \\
& \lambda_{h}^{3} u_{N}^{(3,1, h)}(\xi)=2 \lambda^{3} \gamma^{\frac{3 \alpha^{2}}{4 \pi} h} \sum_{\varepsilon= \pm 1} e^{\alpha^{2} U_{1,2,3}^{(0, h)}(+,-, \varepsilon)} \sum_{h+1}^{N} G_{3,2}^{(i)}(\xi,+-\varepsilon), \\
& \lambda_{h}^{3} u_{N}^{(3,2, h)}(\xi)=2 \lambda^{3}\left(\gamma^{h}\left|\xi_{1}-\xi_{2}\right|\right)^{1-\varepsilon} \gamma^{\frac{3 \alpha^{2}}{4 \pi} h} \\
& \cdot \sum_{\varepsilon= \pm 1}(-\varepsilon) e^{\alpha^{2} U_{1}^{(0, h), 3)}(+,-, \varepsilon)} \sum_{h+1}^{N} G_{32}^{(i)}(\xi,+-\varepsilon), \\
& \lambda_{h}^{4} u_{N}^{(4,1, h)}(\xi)=-\lambda^{4}\left(\gamma^{h}\left|\xi_{1}-\xi_{2}\right|\right)^{2(1-\varepsilon)} \\
& \cdot \sum_{h+1}^{N}\left[6 G_{4,1}^{(i)}(\xi,+-+-)+6 G_{4,2}^{(i)}(\xi,+-+-)\right. \\
& +2 G_{4,3}^{(i)}\left(\xi_{1} \xi_{3} \xi_{2} \xi_{4},+-+-\right)+2 G_{4,4}^{(i)}\left(\xi_{1} \xi_{3} \xi_{2} \xi_{4},+-+-\right) \\
& +2 G_{4,5}^{(i)}\left(\xi_{1} \xi_{3} \xi_{2} \xi_{4},+-+-\right)+4 G_{4,4}^{(i)}(\xi,+-+-) \\
& \left.+2 G_{4,5}^{(i)}(\xi,+-+-)+2 G_{4,5}^{(i)}\left(\xi_{1} \xi_{2} \xi_{4} \xi_{3},+-+-\right)\right], \\
& \lambda_{h}^{4} u_{N}^{(4,2, h)}(\xi)=-\lambda^{4}\left(\gamma^{h}\left|\xi_{3}-\xi_{4}\right|\right)^{2(1-\varepsilon)} \\
& \cdot \sum_{h+1}^{N}\left[6 G_{4,1}^{(i)}(\underline{\xi},+-+-)+6 G_{4,2}^{(i)}(\underline{\xi},+-+-)\right. \\
& +2 G_{4,3}^{(i)}\left(\xi_{1} \xi_{3} \xi_{2} \xi_{4},+-+-\right)+2 G_{4,4}^{(i)}\left(\xi_{1} \xi_{3} \xi_{2} \xi_{4},+-+-\right) \\
& \left.+2 G_{4,5}^{(i)}\left(\xi_{1} \xi_{3} \xi_{2} \xi_{4},+-+-\right)\right] \text {, } \\
& \lambda_{h}^{4} u_{N}^{(4,3, h)}(\underline{\xi})=\lambda^{4}\left(\gamma^{h}\left|\xi_{1}-\xi_{2}\right|\right)^{1-\varepsilon}\left(\gamma^{h}\left|\xi_{3}-\xi_{4}\right|\right)^{1-\varepsilon} \\
& \text { · [all four order terms with } Q=0 \text { ], } \\
& \lambda_{h}^{4} u_{N}^{(4,4, h) N}(\xi)=-\lambda^{4}\left(\gamma^{h}\left|\xi_{1}-\xi_{2}\right|\right)^{2(1-\varepsilon)} 4 \sum_{h+1}^{N} G_{4,3}^{(i)}(\xi,+-+-), \\
& \lambda_{h}^{4} u_{N}^{(4,5, h)}(\xi)=-\lambda^{4}\left(\gamma^{h}\left|\xi_{3}-\xi_{4}\right|\right)^{2(1-\varepsilon)} \\
& \cdot \sum_{h+1}^{N}\left[4 G_{4,3}^{(i)}(\xi,+-+-)+4 G_{4,4}^{(i)}(\xi,+-+-)\right. \\
& \left.+2 \underset{\alpha^{2}}{2 G_{4,5}^{(i)}}(\xi,+-+-)+2 G_{4,5}^{(i)}\left(\xi_{1} \xi_{2} \xi_{4} \xi_{3},+-+-\right)\right], \\
& \lambda_{h}^{4} u_{N}^{(4,6, h)}(\xi)=\lambda^{4} \gamma^{\frac{\alpha^{2}}{\pi} h} \sum_{\varepsilon= \pm 1} e^{\alpha^{2} U_{1,2,3,4}^{(0, h)}(+-\varepsilon \varepsilon)} \sum_{h+1}^{N}\left[4 G_{4,3}^{(i)}(\xi,+-\varepsilon \varepsilon)\right. \\
& \left.+2 G_{44}^{(i)}(\xi,+-\varepsilon \varepsilon)+2 G_{45}^{(i)}(\xi,+-\varepsilon \varepsilon)\right], \\
& \lambda_{h}^{4} u_{N}^{(4,7, h)}(\xi)=\lambda^{4}\left(\gamma^{h}\left|\xi_{1}-\xi_{2}\right|\right)^{1-\varepsilon} \sum_{\varepsilon= \pm 1}(-\varepsilon) e^{\alpha^{2} U_{1,2,3,4}^{(0, h)}(+-\varepsilon \varepsilon)} \\
& \cdot \sum_{h+1}^{N}\left[4 G_{4,3}^{(i)}(\underline{\xi},+-\varepsilon \varepsilon)+2 G_{4,4}^{(i)}(\underline{\xi},+-\varepsilon \varepsilon)+2 G_{4,5}^{(i)}(\underline{\xi},+-\varepsilon \varepsilon)\right] .(\mathrm{A} .28)
\end{aligned}
$$


The bounds (3.14)-(3.16) are a straightforward consequence of some properties of the covariance $\tilde{C}_{0}(x)$ defined in Eq. (2.3). The first property is that there exist $a_{1}, a_{2}, \kappa>0$ such that, $\forall x \in R^{2}$

$$
a_{1} e^{-\kappa|x|} \leqq \tilde{C}_{0}(x) \leqq a_{2} e^{-\kappa|x|} .
$$

Furthermore, given $\eta>0$, there exists $a>0$ such that, $\forall \xi_{1} \xi_{2} \xi_{3} \xi_{4} \in R^{2}$

$$
\begin{gathered}
\left|\tilde{C}_{0}\left(\xi_{1}-\xi_{2}\right)-\tilde{C}_{0}(0)\right| \leqq a\left|\xi_{1}-\xi_{2}\right|^{2-\eta}, \\
\left|\tilde{C}_{0}\left(\xi_{1}-\xi_{3}\right)-\tilde{C}_{0}\left(\xi_{2}-\xi_{3}\right)\right| \leqq a\left|\xi_{1}-\xi_{2}\right|, \\
\left|\tilde{C}_{0}\left(\xi_{1}-\xi_{3}\right)-\tilde{C}_{0}\left(\xi_{2}-\xi_{3}\right)-\tilde{C}_{0}\left(\xi_{1}-\xi_{4}\right)+\tilde{C}_{0}\left(\xi_{2}-\xi_{4}\right)\right| \\
\leqq a\left(\left|\xi_{1}-\xi_{2}\right|\left|\xi_{3}-\xi_{4}\right|\right)^{1-\eta} .
\end{gathered}
$$

Equations (A.3), (A.9), (A.29), and (A.30) are sufficient to show that there exist positive constants $a_{3}, a_{4}, A_{n}$, such that

$$
\begin{aligned}
\left|F^{(h)}\left(X_{1}, \ldots, X_{s} ; \underline{\varepsilon}\right)\right| \leqq & A_{n} \gamma^{-\frac{\alpha^{2}}{4 \pi} h\left(Q^{2}-\sum_{1}^{s} Q_{i}^{2}\right)} e^{-\kappa \gamma^{h} d\left(\underline{\xi}_{X_{1}}, \ldots, \xi_{X_{s}}\right)} \\
& \cdot\left[\prod_{u<v} \prod_{i \in X_{u}} \prod_{j \in X_{v}}\left(1+a_{3}\left|\xi_{i}-\xi_{j}\right|^{a_{4}}\right)\right],
\end{aligned}
$$

where $Q=\sum_{i}^{n} \varepsilon_{i}, Q_{i}=\sum_{j \in X_{i}} \varepsilon_{j}$ and $d\left(\xi_{X_{1}}, \ldots, \xi_{X_{s}}\right)$ is the length of the shortest graph connecting the sets of points $\xi_{X_{1}}, \ldots, \xi_{X_{s}}$. The bound (A.33) can be improved, using Eq. (A.31), if, for some $i,\left|X_{i}\right|=2$ and $Q_{i}=0$; for example, if $X_{1}=\{1,2\}$ and $Q_{1}=0$, we have:

$$
\begin{aligned}
\left|F_{ \pm}^{(h)}\left(X_{1}, \ldots, X_{s} ; \varepsilon\right)\right| \leqq & A_{u}\left(\gamma^{h}\left|\xi_{1}-\xi_{2}\right|\right)^{1+\frac{1 \pm 1}{2}} \gamma^{\frac{\alpha^{2}}{4 \pi} h\left(Q_{i}^{2}-\sum_{1}^{s} Q_{i}^{2}\right)} e^{-\kappa \gamma^{h} d\left(\underline{\mathbf{z}}_{\mathbf{X}_{1}}, \ldots, \underline{\underline{Y}}_{\mathbf{X}_{s}}\right)} \\
& \cdot\left[\prod_{u<v} \prod_{i \in X_{u}} \prod_{j \in X_{v}}\left(1+a_{3}\left|\xi_{i}-\xi_{j}\right|^{a_{4}}\right)\right]
\end{aligned}
$$

where $F_{+}^{(h)}\left(F_{-}^{(h)}\right)$ is obtained from $F^{(h)}$ by symmetrizing (antisymmetrizing) in the exchange $\xi_{1} \leftrightarrow \xi_{2}$.

Finally, using Eq. (A.32), we can prove that

$$
\begin{gathered}
\left|F_{+}^{(h)}((1,2),(3,4) ;+-+-)\right| \leqq A_{n}\left[\left(\gamma^{h}\left|\xi_{1}-\xi_{2}\right|\right)\left(\gamma^{h}\left|\xi_{3}-\xi_{4}\right|\right)\right]^{2(1-\eta)} \\
\cdot\left[\prod_{i \in(1,2)} \prod_{j \in(3,4)}\left(1+a_{3}\left|\xi_{i}-\xi_{j}\right|^{a_{4}}\right)\right] \cdot e^{-\kappa \gamma^{h} d\left(\xi_{(1,2)}, \ldots, \xi_{(3,4)}\right)},
\end{gathered}
$$

where $F_{+}^{(h)}$ is obtained from $F^{(h)}$ by symmetrizing in the exchange $\xi_{1} \leftrightarrow \xi_{2}$ :

The bounds (3.14) $-(3.16)$ are obtained very simply from Eqs. (A.29)-(A.35). In fact, for the functions $u_{N}^{(3,2, h)}$ and $u_{N}^{(4,7, h)}$, Equation (3.14) should be true also if we define them without the factor $\left(\gamma^{h}\left|\xi_{1}-\xi_{2}\right|\right)^{1-\varepsilon}$. This is linked to the fact that the corresponding terms in $\tilde{V}_{I}^{(h)}$ have total charge $Q \neq 0$ and all the terms with $Q \neq 0$ are bounded for any value of the field $\varphi^{(h)}$. This explains the observation following Eq. (3.24).

The positive property (3.17) immediately follows from Eq. (A.19), while the bounds (3.18)-(3.20) are again a straightforward consequence of Eqs. (A.29)(A.35). 


\section{Appendix B}

In this appendix we shall prove the bound (5.13). Let us define:

$$
\Delta_{J}^{(h)}=\left[\sum_{1}^{4} u \frac{1}{u !} \tilde{\mathscr{E}}_{h}^{T}\left(H_{J}^{(h+1)} ; u\right)\right]_{(4)}-\hat{V}_{J}^{(h)} .
$$

From Eqs. (3.23), (3.26), and (A.10)-(A.28) it follows that

$$
\Delta_{J}^{(h)}=-\bar{W}_{2}^{(h)}\left(\mathscr{D}_{h}\right)+\bar{W}_{4}^{(h)}\left(\mathscr{D}_{h}\right)+\bar{C}^{(h)}\left(\mathscr{D}_{h}\right)+\sum_{1}^{4} T_{i}^{(h)}\left(\mathscr{D}_{h}\right),
$$

where

$$
\begin{aligned}
& \bar{W}_{2}^{(h)}\left(\mathscr{D}_{h}\right)=2 \lambda^{2} \int_{\mathscr{D}_{h}} d \xi_{1} d \xi_{2} G_{2,1}^{(h+1)}\left(\xi_{1} \xi_{2} ;+,-\right):\left[1-\cos \alpha\left(\varphi_{\xi_{1}}^{(h)}-\varphi_{\xi_{2}}^{(h)}\right)\right]: \\
& \bar{W}_{4}^{(h)}\left(\mathscr{D}_{h}\right)=-\lambda^{4} \int_{\mathscr{D}_{h} \times J^{2}} d \underline{\xi}_{(1,2)} d \xi_{(3,4)}\left\{:\left[1-\cos \alpha\left(\varphi_{\xi_{1}}^{(h)}-\varphi_{\xi_{2}}^{(h)}\right)\right] \cos \alpha\left(\varphi_{\xi_{3}}^{(h)}-\varphi_{\xi_{4}}^{(h)}\right):\right. \\
& \left.+: \sin \alpha\left(\varphi_{\xi_{1}}^{(h)}-\varphi_{\xi_{2}}^{(h)}\right) \sin \alpha\left(\varphi_{\xi_{3}}^{(h)}-\varphi_{\xi_{4}}^{(h)}\right):\right\} \\
& \cdot\left[6 G_{4,2}^{(h+1)}(\xi,+-+-)+2 G_{4,5}^{(h+1)}\left(\xi_{1}, \xi_{3}, \xi_{2}, \xi_{4},+-+-\right)\right], \\
& T_{1}^{(h)}\left(\mathscr{D}_{h}\right)=-\lambda^{4} \int_{\mathscr{D}_{h} \times J^{c}} d \xi_{(1,2)} d \xi_{(3,4)}\left\{:\left[1-\cos \alpha\left(\varphi_{\xi_{1}}^{(h)}-\varphi_{\xi_{2}}^{(h)}\right)\right] \cos \alpha\left(\varphi_{\xi_{3}}^{(h)}-\varphi_{\xi_{4}}^{(h)}\right):\right. \\
& \left.+: \sin \alpha\left(\varphi_{\xi_{1}}^{(h)}-\varphi_{\xi_{2}}^{(h)}\right) \sin \alpha\left(\varphi_{\xi_{3}}^{(h)}-\varphi_{\xi_{4}}^{(h)}\right):\right\}\left[6 G_{4,1}^{(h+1)}(\xi,+-+-)\right. \\
& \left.+2 G_{4,3}^{(h+1)}\left(\xi_{1}, \xi_{3}, \xi_{2}, \xi_{4},+-+-\right)+2 G_{4,4}^{(h+1)}\left(\xi_{1} \xi_{3} \xi_{2} \xi_{4},+-+-\right)\right] \text {, } \\
& T_{2}^{(h)}\left(\mathscr{D}_{h}\right)=-\lambda^{4} \int_{J^{2} \times \mathscr{D}_{h}} d \underline{\xi}_{(1,2)} d \underline{\xi}_{(3,4)}:\left[1-\cos \alpha\left(\varphi_{\xi_{3}}^{(h)}-\varphi_{\xi_{4}}^{(h)}\right)\right]:\left[6 G_{4,1}^{(h+1)}(\xi,+-+-)\right. \\
& +6 G_{4,2}^{(h+1)}(\xi,+-+-)+2 G_{4,3}^{(h+1)}\left(\xi_{1}, \xi_{3}, \xi_{2}, \xi_{4},+-+-\right) \\
& \left.+2 G_{4,4}^{(h+1)}\left(\xi_{1}, \xi_{3}, \xi_{2}, \xi_{4}\right)+2 G_{4,5}^{(h+1)}\left(\xi_{1}, \xi_{3}, \xi_{2}, \xi_{4},+-+-\right)\right] \text {, } \\
& T_{3}^{(h)}\left(\mathscr{D}_{h}\right)=-\lambda^{4} \int_{J^{2} \backslash \mathscr{D}_{h} \times \mathscr{D}_{h}} d \xi_{(1,2)} d \xi_{(3,4)}:\left[1-\cos \alpha\left(\varphi_{\xi_{3}}^{(h)}-\varphi_{\xi_{4}}^{(h)}\right)\right]:\left[4 G_{4,4}^{(h+1)}(\xi,+-+-)\right. \\
& \left.+2 G_{4,5}^{(h+1)}(\xi,+-+-)+2 G_{4,5}^{(h+1)}\left(\breve{\zeta}_{1}, \breve{\zeta}_{2}, \breve{\zeta}_{4}, \breve{\zeta}_{3},+-+-\right)\right], \\
& T_{4}^{(h)}\left(\mathscr{D}_{h}\right)=\lambda^{4} \int_{J^{2} \backslash \mathscr{D}_{h} \times \mathscr{D}_{h}} d \underline{\xi}_{(1,2)} d \underline{\xi}_{(3,4)}: \sin \alpha\left(\varphi_{\xi_{1}}^{(h)}-\varphi_{\xi_{2}}^{(h)}\right) \sin \alpha\left(\varphi_{\xi_{3}}^{(h)}-\varphi_{\xi_{4}}^{(h)}\right): \\
& \cdot\left\{6 G_{4,1}^{(h+1)}(\underline{\xi},+-+-)+6 G_{4,2}^{(h+1)}(\underline{\xi},+-+-)\right. \\
& +2 G_{4,3}^{(h+1)}\left(\xi_{1}, \xi_{3}, \xi_{2}, \xi_{4},+-+-\right)+2 G_{4,4}^{(h+1)}\left(\xi_{1}, \xi_{3}, \xi_{2}, \xi_{4},+-+-\right) \\
& +2 G_{4,5}^{(h+1)}\left(\xi_{1}, \xi_{3}, \xi_{2}, \xi_{4},+-+-\right)+4 G_{4,4}^{(h+1)}(\underline{\xi},+-+-) \\
& \left.+2 G_{4,5}^{(h+1)}(\xi,+-+-)+2 G_{4,5}^{(h+1)}\left(\xi_{1}, \xi_{2}, \xi_{4}, \xi_{3},+,-,+,-\right)\right\}, \\
& \bar{C}^{(h)}\left(\mathscr{D}_{h}\right)=-\lambda^{4} \int_{\mathscr{D}_{h} \times J^{2}} d \underline{\xi}_{(1,2)} d \underline{\xi}_{(3,4)}\left[4 G_{4,4)}^{(h+1)}(\underline{\xi},+-+-)\right. \\
& \left.+2 G_{4,5}^{(h+1)}(\xi,+-+-)+2 G_{4,5}^{(h+1)}\left(\xi_{1}, \xi_{2}, \xi_{4}, \xi_{3},+-+-\right)\right] \\
& -\lambda^{4} \int_{\left(\mathscr{D}_{h} \times J^{2}\right) \cup\left(J^{2} \times \mathscr{D}_{h}\right)} d \underline{\xi}_{(1,2)} d \underline{\xi}_{(3,4)} 4 G_{4,3}^{(h+1)}(\underline{\xi},+-+-) .
\end{aligned}
$$


Using Eqs. (A.29)-(A.35), it is easy to show that there exists $A>0$ such that

$$
\left|\sum_{i}^{4} T_{i}^{(h)}\left(\mathscr{D}_{h}\right)\right| \leqq A B_{h} \lambda_{h}^{2} \bar{W}_{2}^{(h)}\left(\mathscr{D}_{h}\right) .
$$

Furthermore, if $B$ is large enough, $\forall h \geqq 0$ :

$$
\bar{W}_{2}^{(h)}\left(\mathscr{D}_{h}\right) \geqq 0
$$

Equations (B.10) and (B.11) imply that there exist $\tilde{h}(\bar{B}, \lambda)$ and $\bar{B}_{0}$ such that, if $\bar{B} \geqq \bar{B}_{0}$ (recall that $\left.B=\sigma \bar{B}\right)$ and $h \geqq \tilde{h}(\bar{B}, \lambda)$ :

$$
\Delta_{J}^{(h)} \leqq-\frac{1}{2} \bar{W}_{2}^{(h)}\left(\mathscr{D}_{h}\right)+\bar{W}_{4}^{(h)}\left(\mathscr{D}_{h}\right)+\bar{C}^{(h)}\left(\mathscr{D}_{h}\right) .
$$

The point now is that $\bar{W}_{4}^{(h)}\left(\mathscr{D}_{h}\right)$ and $\bar{C}^{(h)}\left(\mathscr{D}_{h}\right)$ do not satisfy a bound like (B.10) uniformly in $N$. Then it is not clear if $\Delta_{J}^{(h)}$ is negative for any choice of $\mathscr{D}_{h}$, or not. We did not solve this problem; however we can prove that $\Delta_{J}^{(h)}$ is bounded by something which is summable in $h$, at least for $\alpha^{2}$ small enough (but larger than $6 \pi)$. Of course, if $\Delta_{J}^{(h)}$ were negative, the proof of Lemma 2 would be finished without any restriction on $\alpha^{2}$.

Let us define

$$
D_{a, \eta}^{(h)}=\left\{\left(\xi_{1}, \xi_{2}\right) \in I^{2}|| \xi_{1}-\xi_{2} \mid \leqq \gamma^{-a h} \lambda^{\eta}\right\}
$$

It is easy to show that there exists $c>0$ such that

$$
\begin{aligned}
& \left|\bar{W}_{4}^{(h)}\left(\mathscr{D}_{h} \cap D_{a, \eta}^{(h)}\right)\right| \leqq c \lambda^{4+\left(4-\frac{\alpha^{2}}{2 \pi}\right) \eta}|I| \gamma^{\left[\left(\frac{\alpha^{2}}{2 \pi}-2\right)+a\left(\frac{\alpha^{2}}{2 \pi}-4\right)\right] h} \\
& \left|\bar{C}^{(h)}\left(\mathscr{D}_{h} \cap D_{a, \eta}^{(h)}\right)\right| \leqq c \lambda^{4+\left(4-\frac{\alpha^{2}}{2 \pi}\right) \eta}|I| \gamma^{\left[\left(\frac{\alpha^{2}}{2 \pi}-2\right)+a\left(\frac{\alpha^{2}}{2 \pi}-4\right)\right] h} \\
& \left|\bar{W}_{4}^{(h)}\left(\mathscr{D}_{h} \backslash D_{a, \eta}^{(h)}\right)\right| \leqq c \lambda^{2-\left(\frac{\alpha^{2}}{2 \pi}-2\right) \eta} \gamma^{-h\left[2-a\left(\frac{\alpha^{2}}{2 \pi}-2\right)\right]} \bar{W}_{2}^{(h)}\left(\mathscr{D}_{h} \backslash D_{a, \eta}^{(h)}\right) \\
& \left|\bar{C}^{(h)}\left(\mathscr{D}_{h} \backslash D_{a, \eta}^{(h)}\right)\right| \leqq c \lambda^{2-\frac{\alpha^{2}}{2 \pi} \eta} \gamma^{-h\left[4-\frac{\alpha^{2}}{2 \pi} a\right]} \bar{W}_{2}^{(h)}\left(\mathscr{D}_{h} \backslash D_{a, \eta}^{(h)}\right)
\end{aligned}
$$

From these bounds and Eq. (B.12), it follows that, if

$$
a<\frac{8 \pi}{\alpha^{2}}, \quad \eta<\frac{4 \pi}{\alpha^{2}},
$$

there exists $\bar{h}(\bar{B}, \lambda) \geqq \tilde{h}(\bar{B}, \lambda)$ such that, if $h>\bar{h}(\bar{B}, \lambda)$

$$
\Delta_{J}^{(h)} \leqq 2 c \lambda^{4\left[1+\eta\left(1-\frac{\alpha^{2}}{8 \pi}\right)\right]}|I| \gamma^{-\varrho h},
$$

where

$$
\varrho=\left(4-\frac{\alpha^{2}}{2 \pi}\right) a-\left(\frac{\alpha^{2}}{2 \pi}-2\right)
$$


and $\bar{h}(\bar{B}, \lambda) \underset{\lambda \rightarrow 0}{\longrightarrow} 0$. Since we want $\varrho>0$, we have to impose on $a$ the condition

$$
a>\frac{\frac{\alpha^{2}}{2 \pi}-2}{4-\frac{\alpha^{2}}{2 \pi}},
$$

which is compatible with (B.18) only if

$$
\alpha^{2}<2 \pi(\sqrt{17}-1)
$$

\section{Appendix C}

In this appendix we show how it is possible to reduce the proof of Lemma 1 to the proof of Lemma 1 in [2], which the reader is supposed to be familiar with.

Let $Q_{1}, Q_{2}, Q_{3}$ be three pavements of $R$ with cubic tesserae with side size 1 . We suppose that the centers of the tesserae of such pavements are on the step -1 lattices of $R^{2}$ with origin, respectively, at the points

$$
\bar{\xi}_{k}=\frac{2^{k-1}-1}{2^{k-1}}(1,1) \quad k=1,2,3
$$

and with edges parallel to the coordinate axes of $R^{2}$. After constructing such pavements we turn each tessera into a smooth region by some deformations, so that different tesserae are separated by a corridor and the intersections between the boundaries are smooth. The exact definition of the deformed tesserae can immediately be deduced by the analogous definition in Sect. 5 of [2], where the three-dimensional case is treated. We call $\tilde{Q}_{1, \ell}, \tilde{Q}_{2, \ell}, \tilde{Q}_{3, \ell}$ the sets of deformed tesserae, scaled by a homothety factor $\ell$. On each $\square \in \tilde{Q}_{i, \ell}, i=1,2,3$, we introduce a covering of $\partial \square$ with regular line-elements, regularly spaced as $\ell \rightarrow \infty$ (see Appendix A of [2], Definition 3), which will be denoted $\sigma_{1}, \sigma_{2}, \ldots$ To each line element we associate its local system of coordinates (see Appendix A of [2], Definition 3 and Fig. 1). If $f$ is a distribution in $\mathscr{D}^{\prime}(\partial \square)$ and if $\alpha_{\sigma_{1}}, \alpha_{\sigma_{2}}, \ldots$, is a partition of unity on $\partial \square$ associated with the regular, regularly spaced covering, we consider the distribution $\alpha_{\sigma} f$ with support on $\sigma$ and call $\overline{\alpha_{\sigma} f}$ its representative in the local system of coordinates associated with $\sigma: \overline{\alpha_{\sigma} f} \in \mathscr{D}^{\prime}\left(R^{2}\right)$

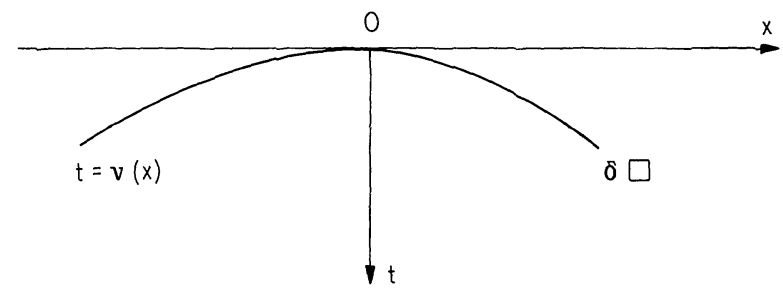

Fig. 1 
The following norms will be used, $\forall s \in R, \forall \varepsilon \in(0,1)$ :

$$
\begin{aligned}
& \|f\|_{\mathscr{C}(\xi)(\sigma)}=\left\|\overline{\alpha_{\sigma} f}\right\|_{\mathscr{C}_{(\xi)}(R)}, \\
& \|g\|_{\mathscr{C}_{S}^{(\varepsilon)}(R)}=\left\|\left(1-\partial_{\mathbf{x}}^{2}\right)^{\frac{s-\varepsilon}{2}} g\right\|_{\bar{C}^{(\varepsilon)}(R)}, \\
& \|h\|_{\bar{C}^{(\varepsilon)}(G)} \sup _{x \in G} e^{V|x|}|h(x)|+\sup _{\substack{x, y \in G \\
|x-y| \leqq 1}} e^{V \sqrt{|x|}} \frac{|h(x)-h(y)|}{|x-y|^{\varepsilon}}, \quad G \in R^{d} .
\end{aligned}
$$

We shall need the following proposition

Proposition C.1. If $0<\varepsilon<s<1$, there exists a constant $c_{s, \varepsilon}$ such that, for any $u \in \mathscr{C}_{s}^{(\varepsilon)}(R)$

where

$$
\|u\|_{\bar{C}^{(x)}(\sigma)} \leqq c_{s, \varepsilon}\|u\|_{\mathscr{C}_{s}^{(\varepsilon)}(R)},
$$

$$
\alpha=s-\varepsilon .
$$

Proof. If $u \in \mathscr{C}_{s}^{(\varepsilon)}(R)$, there is a function $z \in \bar{C}^{(\varepsilon)}(R)$ such that

$$
u(x)=\int_{-\infty}^{+\infty} N_{\alpha}(x-y) z(y) d y
$$

where $N_{\alpha}(x-y)$ is the Kernel of $\left(1-\partial_{x}^{2}\right)^{-\alpha / 2} . N_{\alpha}(x)$ has the following properties:

$$
\begin{aligned}
& N_{\alpha}(x)=|x|^{\alpha-1} I\left(x^{2}\right)+J\left(x^{2}\right) \quad|x| \leqq 1, \\
& \left|\partial_{x}^{p} N_{\alpha}(x)\right| \leqq c_{p} e^{-\kappa|x|}, \quad \forall p \geqq 0, \quad|x| \geqq 1,
\end{aligned}
$$

where $I\left(\mathbf{x}^{2}\right), J\left(\mathbf{x}^{2}\right)$ are smooth functions and $\kappa>0$ is a suitable constant. (C.5) is an easy consequence of (C.8), (C.9) and the estimate:

$$
\int_{|y| \leqq 1} d y|| x_{1}-\left.y\right|^{\alpha-1}-\left|x_{2}-y\right|^{\alpha-1}|\leqq c| x_{1}-\left.x_{2}\right|^{\alpha}
$$

valid for any $x_{1}, x_{2} \in[-1 / 2,1 / 2]$ and a suitable $c>0$. If $\alpha_{\sigma}$ belongs to the partition of unity on $\partial \square$ defined before, we call $\tilde{\alpha}_{\sigma}$ an extension of $\alpha_{\sigma}$ to a $C^{\alpha}$ function on $R^{2}$ with support within distance 1 from $\sigma$, chosen in a "canonical" way (see [4, Sect. 2]). Then, if $u \in \mathscr{S}^{\prime}\left(R^{2}\right)$ we define:

$$
\zeta_{\sigma}^{(J)}(x, t)=\alpha_{\sigma}(x, t+\gamma(x))\left(\partial^{(J)} u\right)(x, t+\gamma(x)), \quad J=0,1,
$$

where $t=\gamma(x)$ describes the surface element $\sigma$ in the local coordinates (see Fig. 1) and $\partial^{(J)}$ denotes the normal derivative of order $J$. We put also

$$
\theta_{\sigma}^{(J)}(x, t)=\left(1-\partial_{x}^{2}\right)^{\frac{s-J-\varepsilon}{2}} \zeta_{\sigma}^{(J)}(x, t), \quad J=0,1
$$

for any $s \in R$ and $\varepsilon \in(0,1)$. Then we define (see [4, Sect. 2]), for $G=R^{2}$ or $G=\square \in \bigcup_{i, \ell} \tilde{Q}_{i, \ell}$ and $G^{\prime}=R^{2}$ or $R \times[0, \infty)$ respectively :

$$
\begin{aligned}
\|u\|_{\mathscr{C}(\xi)(G, \sigma)} & =\sum_{0}^{1}\left\|\theta_{\sigma}^{(J)}\right\| \bar{c}^{(\varepsilon)}\left(G^{\prime}\right), \\
\|u\|_{\mathscr{C}_{s}^{(\varepsilon)}(G, \partial \square)} & =\sup _{i}\|u\|_{\mathscr{C}_{s}^{(\varepsilon)}\left(G, \sigma_{i}\right)},
\end{aligned}
$$

where, in Eq. (C.14), the supremum is taken on all the surface elements of $\partial \square$. 
We shall need the following proposition

Proposition C.2. If $0<\varepsilon<s<1$, there exists two constants $\Gamma_{\varepsilon, s}$ and $\delta$ such that, if $\ell_{0}$ is large enough, for any $\square \in \bigcup_{\ell \geqq \ell_{0}} \bigcup_{i} \tilde{Q}_{i, \ell}$

$$
\|u\|_{C^{(s-\varepsilon)}(S \square)} \leqq \Gamma_{\varepsilon, s}\|u\|_{\mathscr{C}(\varepsilon)(\square, \partial \square)},
$$

where

$$
S_{\square}=\{\underline{\xi} \in \square \mid d(\underline{\xi}, \partial \square) \leqq \delta\} .
$$

Proof. By the "canonical" definition of the functions $\tilde{\alpha}_{\sigma}$ (see [4, Sect. 2]), it is possible to choose $\delta$, at least for $\ell$ large enough, so that, if $\underline{\xi} \in S_{\square}, \tilde{\alpha}_{\sigma}(\xi) \geqq c$ for a suitable $\sigma \subset \partial \square$, with $c$ independent of $\sigma$. Then the proof of Proposition 2 easily follows from the following lemma (the independence of $\Gamma_{\varepsilon, s}$ on $\ell$ is a simple consequence of the exact definition of $\alpha_{\sigma}$ ).

Lemma 3. Let $u(x, t)$ be a function defined on the half-plane $t \geqq 0$, such that

$$
\begin{gathered}
u(x, t)=\left(1-\partial_{\mathbf{x}}^{2}\right)^{-\frac{\alpha}{2}} \theta_{0}(x, t), \\
\partial_{t} u(x, t)=\left(1-\partial_{\mathbf{x}}^{2}\right)^{-\frac{\alpha-1}{2}} \theta^{1}(x, t),
\end{gathered}
$$

where $\partial_{t} u$ denotes the derivative in the sense of the distributions and

$$
\begin{gathered}
\theta_{i} \in \bar{C}^{(\varepsilon)}(R \times[0, \infty), \quad i=0,1, \\
0<\alpha<1, \quad \varepsilon \geqq 0 .
\end{gathered}
$$

Then there exists a constant $c$, such that

$$
\|u\|_{C^{(\alpha)}(R \times[0, \infty))} \leqq c \sum_{0}^{1}\left\|\theta_{i}\right\|_{\bar{C}^{(\varepsilon)}(R \times[0, \infty))} .
$$

Proof. By Prop. C.1 and Eq. (C.16), $\forall x_{1}, x_{2} \in R$ :

$$
\left|u\left(x_{1} t\right)-u\left(x_{2} t\right)\right| \leqq c\left\|\theta_{0}\right\|\left|x_{1}-x_{2}\right|^{\alpha}
$$

for a suitable $c$, uniformly in $t \geqq 0$. Then it is sufficient to show that $u(x, t)$ is Hölder continuous in $t$ of order $\alpha$, uniformly in $x \in R$. Let $\chi(x)$ be a $C^{\infty}$ function such that $\operatorname{supp} \chi=[-1,1], \chi \geqq 0$, and $\int \chi(x) d x=1$. If $\mu=t_{2}-t_{1}>0$, we define $\chi_{\mu}(x)=\frac{1}{\mu} \chi\left(\frac{x}{\mu}\right)$; then we can write:

$$
\begin{aligned}
u\left(x, t_{2}\right)-u\left(x, t_{1}\right)= & \int \chi_{\mu}(\delta) d \delta\left[u\left(x, t_{2}\right)-u\left(x+\delta, t_{2}\right)\right] \\
& +\int \chi_{\mu}(\delta) d \delta\left[u\left(x+\delta, t_{2}\right)-u\left(x+\delta, t_{1}\right)\right] \\
& +\int \chi_{\mu}(\delta) d \delta\left[u\left(x+\delta, t_{1}\right)-u\left(x, t_{1}\right)\right] .
\end{aligned}
$$


By Eqs. (C.21) and (C.17), we have

$$
\begin{aligned}
& \left|u\left(x, t_{2}\right)-u\left(x, t_{1}\right)\right| \leqq 2 c\left\|\theta_{0}\right\| \int \delta^{\alpha} \chi_{\mu}(\delta) d \delta
\end{aligned}
$$

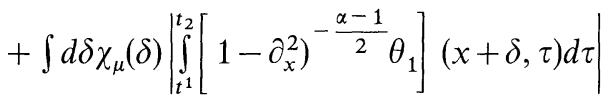

$$
\begin{aligned}
& \leqq 2 c\left\|\theta_{0}\right\| \mu^{\alpha} \int x^{\alpha} \chi(x) d x \\
& +\mu\left\|\theta_{1}\right\| \int d x\left|\left(1-\partial_{x}^{2}\right)^{-\frac{(\alpha-1)}{2}} \chi_{\mu}(x)\right| .
\end{aligned}
$$

Then it is sufficient to show that:

$$
\begin{aligned}
\mu \int d x \mid\left(1-\partial_{x}^{2}\right)^{-\frac{(\alpha-1)}{2}} \chi_{\mu}(x) & =(2 \pi)^{-1 / 2} \mu^{\alpha} \int d x\left|\int d h e^{i h y} \tilde{\chi}(h)\left(\mu^{2}+h^{2}\right)^{\frac{1-\alpha}{2}}\right| \\
& \leqq \cos \mu^{\alpha} .
\end{aligned}
$$

Equation (C.24) can be easily proven by standard calculations.

Let us now consider the elliptic operator

$$
A=\left(\gamma^{2}-1\right)(1-D)\left(\gamma^{2}-D\right),
$$

where $D$ is the Laplacian on $R^{2}$. In the theory of $A$ a "canonical regularity" parameter, $\Theta(\gamma)$, appears which tends to 0 as $\gamma \rightarrow 1$ (see [4, Sect. 2]). We shall therefore choose the value of $\gamma$, so far arbitrary, so close to 1 that all the tesserae $\square \in \bigcup_{i, \ell} \tilde{Q}_{i, \ell}$ are conically regular with respect to the cones with opening $\Theta(\gamma)$. We are interested in the Dirichlet problem:

$$
\begin{gathered}
A u=0 \text { in } \square \\
\partial^{(J)} u=z^{(J)} \text { on } \partial \square, \quad J=0,1 .
\end{gathered}
$$

It is convenient to introduce the "space of boundary data"

$$
\mathfrak{C}_{s}^{(\varepsilon)}(\partial \square)=\prod_{J=0}^{1} \mathscr{C}_{s-J}^{(\varepsilon)}(\partial \square)
$$

where we used the definition:

$$
\|f\|_{\mathscr{C}(\varepsilon)(\partial \square)}=\sup _{i}\|f\|_{\mathscr{C}(\xi)\left(\sigma_{i}\right)} .
$$

If $\underline{z}=\left(z^{(0)}, z^{(1)}\right) \in \mathfrak{C}_{s}^{(\varepsilon)}(\partial \square)$, we put also

$$
\|\underline{Z}\|_{\mathfrak{C}(\varepsilon)\left(\sigma_{i}\right)}=\sum_{0}^{1}\left\|z^{(J)}\right\|_{\zeta_{\zeta}^{(\varepsilon)} J\left(\sigma_{i}\right)}
$$

The following proposition contains what we need to solve the problem (C.26)

Proposition C.4. There exist positive constants $\ell_{0}, \kappa, c_{s, \varepsilon}$ such that, if $\ell \geqq \ell_{0}$ and $\underline{z} \in \mathfrak{C}_{s}^{(\varepsilon)}(\partial \square)$, the problem (C.26) has a unique solution with the following properties:

(i) $\forall s \in R, \forall \varepsilon \in(0,1)$, if $s^{\prime}<s$,

$$
\|u\|_{\mathscr{C}_{\left.S^{(}\right)(\square, \partial \square)} \leqq} \bar{c}_{s, s^{\prime}, \varepsilon}\|\underline{z}\|_{\mathbb{C}(\xi)(\partial \square)}
$$


for a suitable constant $\bar{c}_{s, s^{\prime}, \varepsilon}$,

(ii) if $0<\varepsilon<s<1, \forall \Delta \subset \square, \Delta$ open, $\forall \sigma_{i} \subset \partial \square$

$$
\|u\|_{C^{\left(s^{-\varepsilon}\right)(\Delta)}} \leqq c_{s, \varepsilon} e^{-\kappa d\left(\Delta, \sigma^{i}\right)}\|\underline{z}\|_{\mathfrak{C}_{(\xi)\left(\sigma^{l}\right)}},
$$

(iii) if $\sigma_{J}^{\prime} \in \square^{\prime}, \sigma_{J}^{\prime} \subset \bar{\square}, \forall s \in R, \forall \varepsilon \in(0,1), \forall s^{\prime}<s$

$$
\|\underline{\partial} u\|_{\mathfrak{C}_{S^{\prime}\left(\sigma^{\prime}\right)}\left(\sigma_{J}^{\prime}\right)} \leqq \bar{c}_{s, s^{\prime}, \varepsilon} e^{-\kappa d\left(\sigma_{J}^{\prime}, \sigma_{i}\right)}\|\underline{z}\|_{\mathfrak{C}(\varepsilon)\left(\sigma_{l}\right)},
$$

where $\underline{\partial} u=\left(\partial^{(0)} u, \partial^{(1)} u\right)$ denotes the traces of $u$ and $\partial^{(1)} u$ on $\partial \square^{\prime}$.

Proof. The points (i) and (iii) immediately follow from Prop. 1 of [4], where it is also proven that

$$
\|u\|_{C^{(\varepsilon)}(\Delta)} \leqq c_{s, \varepsilon} e^{-\kappa d\left(\Delta, \sigma_{i}\right)}\|\underline{z}\|_{\mathscr{C}(\varepsilon)\left(\sigma_{l}\right)} .
$$

Since $u(\xi)$ is a $C^{\infty}$ function for $\xi \in \square \backslash S_{\square}$, Prop. 2 and Eq. (C.33) imply Eq. (C.31).

Let now $P$ be the gaussian process on $\mathscr{S}^{\prime}\left(R^{2}\right)$ whose covariance operator is $A^{-1}$ [cf. Eq. (2.3)]. There is a support property for $P$, which plays an essential role in the proof of Lemma 1 . Call $\Sigma_{i, \ell} i=1,2,3$ the family of surface elements of $\partial \tilde{Q}_{i, \ell}$ and call $\Sigma_{\ell}=\bigcup_{1}^{3} \Sigma_{i, \ell}$. Given a square $I$ centered at the origin, let:

$$
\bar{E}_{\sigma}^{B, s, \eta, \ell}=\left\{z \in \mathscr{S}^{\prime}\left(R^{2}\right) \mid\|\underline{\partial} z\|_{\mathbb{C}_{(\eta)}(\sigma)}<B_{\sigma}\right\},
$$

where $\left.\sigma \in \Sigma_{\ell}, B_{\sigma}=B\left(1+d \mid \sigma_{1} I\right)\right)$ and $\underline{\partial z}=\left(\partial^{(0)} z, \partial^{(1)} z\right)$ denotes the traces of $z$ and $\partial^{(1)} z$ on $\sigma$.

Let $\bar{\chi}_{\sigma}^{B, \mathbf{s}, \eta, \ell}$ be the characteristic function of $\bar{E}_{\sigma}^{B, \mathbf{s}, \eta, \ell}$, which we shall abbreviate $\bar{\chi}_{\sigma}^{B, \mathbf{s}, \ell}$ or $\bar{\chi}_{\sigma}^{B, \mathbf{s}}$ or $\bar{\chi}_{\sigma}^{B}$. Then :

Proposition C.5. Fix $\eta \in(0,1 / 2)$ and $s<1, \eta<s$. There exist constants $c_{i}: i=1, \ldots, 6$ and $\ell_{1}$ such that, if $\ell \geqq \ell_{1}$

(i) $\forall B \geqq c_{3}+c_{4} \log \ell$

$$
\int P(d z) \prod_{\sigma \in \Sigma_{l}} \bar{\chi}_{\sigma}^{B, s} \geqq \exp \left\{-c_{1} e^{-c_{2} B^{2}}|I|\right\} .
$$

(ii) If $S \subset \Sigma_{\ell}, \forall B_{\sigma} \geqq c_{3}+c_{4} \log \ell, \sigma \in S$

$$
\int P(d z) \prod_{\sigma \in S}\left(1-\bar{\chi}_{\sigma}^{B, s}\right) \leqq \prod_{\sigma \in S} \exp \left(c_{5}-c_{6} B_{\sigma}^{2}\right) .
$$

(iii) Let $\chi_{\Delta}^{B}$ be the characteristic function of the event

$$
E_{\Delta}^{B}=\left\{z \in \mathscr{S}^{\prime}\left(R^{2}\right) \mid\|z\|_{C^{(s-\eta)(\Delta)}}<B(1+d(\Delta, I))\right\},
$$

where $\Delta \in Q_{1}$, the pavement made of square tesserae of size 1 . Then, if $B_{\Delta}=B(1+d(\Delta, I))$ :

$$
\int P(d z)\left(\prod_{\Delta \in Q_{1}} \chi_{\Delta}^{B}\right)\left(\prod_{\sigma \equiv \Sigma} \bar{\chi}_{\sigma}^{B}\right) \geqq \exp \left(-c_{1} e^{-c_{2} B^{2}}|I|\right),
$$

$\forall B \geqq \mathbf{c}_{3}+\mathbf{c}_{4} \log \ell$ and, if $\mathscr{R} \subset Q_{1}$ :

$$
\int P(d z)\left[\prod_{\Delta \in \mathscr{R}}\left(1-\chi_{\Delta}^{B}\right)\right] \leqq \prod_{\Delta \in \mathscr{R}} \exp \left(c_{5}-c_{6} B_{\Delta}^{2}\right) .
$$


(iv) The estimates (C.36) and (C.39) hold also if $P$ is replaced by the probability measure $P_{\square}^{0}$, associated with the operator $A$ considered with zero boundary conditions on $\partial \square$.

Proof. Proposition C.5 is essentially a particular case of Propositions 3 and 4 of [4]. The only difference is in the estimates (C.38) and (C.39), which were proven in [4] with $\eta$ instead of $s-\eta$ in the definition (C.37) of $E_{\Delta}^{B}$.

If $s-\eta>\eta$, the stronger result of Proposition C. 5 follows from Proposition C.4 (where the stronger bound (C.31) replaces the bound (C.33) used in [4]) and from the following simple property of the covariance $\tilde{C}_{0}=A^{-1}$ :

$$
\left|\tilde{C}_{0}(x)-\tilde{C}_{0}(0)\right| \leqq c_{\varepsilon}|x|^{2-2 \varepsilon}
$$

for any $\varepsilon>0$ and a suitable constant $c_{\varepsilon}$. Equation (C.40) implies in fact that $P$ and $P_{\square}^{0}$ have support on the functions, which are Hölder-continuous of order $1-\varepsilon$. Then the proof goes exactly like in Sects. 5-7 of [4], choosing $\varepsilon$ so that $s-\eta=1-\varepsilon$.

Remark. The conditions on $s$ and $\eta$ allow us to make $s-\eta=1-\varepsilon$ with $\varepsilon$ arbitrary small. This result does not follow from [4], which would allow us to prove Lemma 1 only for $\varepsilon>1 / 2$. At this point the proof of Lemma 1 can be continued repeating word by word, with some obvious changes, the arguments of [2] which, there, follow Proposition 2 in Sect. 5.

\section{References}

1. Fröhlich, J.: Commun. Math. Phys. 47, 233 (1976)

Albeverio, S., Høegh-Krohn, R.: Commun. Math. Phys. 30, 171 (1973)

Edwards, S., Lenard, A. : J. Math. Phys. 3, 778 (1962)

Thacker, H.B.: Rev. Mod. Phys. 53, 253-286 1981

2. Benfatto, G., Cassandro, M., Gallavotti, G., Nicolò, F., Olivieri, E., Presutti, E., Scacciatelli, E. : Commun. Math. Phys. 71, 95 (1980)

3. Gallavotti, G.: Mem. Accad. Lincei 15, 23 (1978)

Gallavotti, G. : Ann. Mat. Pura Appl. 70, 1 (1979)

Benfatto, G., Cassandro, M., Gallavotti, G., Nicolò, F., Olivieri, E., Presutti, E., Scacciatelli, E.: Commun. Math. Phys. 59, 143 (1978)

4. Benfatto, G., Gallavotti, G., Nicolò, F.: J. Funct. Anal. 36, 343 (1980)

5. Sklyapin, L.D., Taktadian, L.A., Faddeev, L.D. : Teor. Mat. Fiz. 40, 194-220 (1979)

Communicated by K. Osterwalder 